\title{
Diversidade de anfíbios e répteis Squamata na região do baixo rio Purus, Amazônia Central, Brasil
}

\author{
Fabiano Waldez ${ }^{1,2,5}$, Marcelo Menin ${ }^{3}$ \& Richard Carl Vogt ${ }^{4}$ \\ ${ }^{1}$ Programa de Pós-graduação em Ecologia, Instituto Nacional de Pesquisas da Amazônia - INPA, CP 478, \\ CEP 69011-970, Manaus, AM, Brasil. http://www.inpa.gov.br \\ ${ }^{2}$ Instituto Federal de Educação, Ciência e Tecnologia do Amazonas - IFAM, Campus Tabatinga, CP 67, \\ CEP 69640-000, Tabatinga, AM, Brasil. http://www.ctb.ifam.edu.br \\ ${ }^{3}$ Departamento de Biologia, Instituto de Ciências Biológicas, Universidade Federal do Amazonas - UFAM, \\ Av. General Rodrigo Otávio Jordão Ramos, 3000, \\ CEP 69077-000, Manaus, AM, Brasil. http://portal.ufam.edu.br \\ ${ }^{4}$ Curador da Coleção de Anfíbios e Répteis, Instituto Nacional de Pesquisas da Amazônia - INPA, \\ Av. André Araújo, 2936, Aleixo, CEP 69060-001, Manaus, AM, Brasil. http://www.inpa.gov.br \\ ${ }^{5}$ Autor para correspondência: Fabiano Waldez, e-mail: fwaldez@yahoo.com.br
}

WALDEZ, F., MENIN, M. \& VOGT, R.C. Diversity of amphibians and Squamata reptilians from lower Purus River Basin, Central Amazonia, Brazil. Biota Neotrop. 13(1): http://www.biotaneotropica.org.br/v13n1/ en/abstract?inventory+bn03113012013

\begin{abstract}
The lower Purus River Basin, Central Amazonia - Brazil, represents an area of prominent interest for conservation of amphibians and reptiles. However, there is little available information about these groups for flooded and nonflooded forests, the major landscapes in this region. We sampled a representative area over two periods: 2004-2005 and 2009-2010. We used efficient and complementary techniques for sampling the herpetofauna in rainforests: time constrained audio-visual search (TAVS), pitfall traps with drift fence (PFDF) and doubleended funnel traps with drift fence (FTDF). We recorded a total of 160 species, including 75 amphibians (73 frogs and two caecilians) and 85 reptiles (34 lizards and 51 snakes). The occurrence of the anuran Dendropsophus allenorum represents the first record for Brazil. Comparing the efficiency of the sampling methods, the TAVS was the best method in sampling efficiency for the majority of the herpetofauna species; followed by the PFDF, efficient in sampling terrestrial frogs and litter lizards in the nonflooded forests. We did not use the PFDF in the flooded forests because the soil was water saturated. Despite its low efficiency in sampling terrestrial frogs and litter lizards, the FTDF, was efficient in snake capture and easily adaptable to the saturated soil in flooded forests. The greatest richness of species and groups was found in the nonflooded forests. Families of fossorial and litter species were mostly absent from the flooded forests. However, in the flooded forests we found a large number of arboreal frogs (Hylidae) and of larger heliothermic lizards (Teiidae). When we evaluated each sample period separately, we found a similar richness of the herpetofauna between the different types of forest landscapes, with a bias towards higher diversity in the nonflooded forests. In spite of similar species numbers, the nonflooded and flooded forests had different species assemblages. The total amphibian and Squamata reptilian diversity between the nonflooded and flooded forests (beta- $\beta$ diversity), sites with more widespread landscapes, possibly represents the major contribution to the regional herpetofauna diversity in the lower Purus River Basin (Gama- $\gamma$ diversity). Keywords: Brazilian Amazon, flooded and nonflooded forests, species distribution, herpetofauna.
\end{abstract}

WALDEZ, F., MENIN, M. \& VOGT, R.C. Diversidade de anfíbios e répteis Squamata na região do baixo rio Purus, Amazônia Central, Brasil. Biota Neotrop. 13(1): http://www.biotaneotropica.org.br/v13n1/pt/abst ract?inventory+bn03113012013

Resumo: A região do baixo rio Purus na Amazônia Central - Brasil representa uma área de relevante interesse para conservação de anfíbios e répteis. No entanto, há pouca informação disponível sobre estes grupos taxonômicos para áreas de florestas de terra firme e florestas de várzea, principais paisagens nesta região. Nesse estudo, realizamos uma amostragem ampla em esforço e área, em dois períodos: 2004-2005 e 2009-2010. Utilizamos técnicas complementares eficientes na amostragem da herpetofauna em florestas tropicais: procura ativa limitada por tempo (PLT), armadilhas de interceptação e queda (AIQ) e armadilhas de interceptação com funis duplos (AIF). Foram registradas 160 espécies, sendo 75 de anfíbios (73 anuros e dois gimnofionos) e 85 de répteis (34 lagartos e 51 serpentes). O registro do anuro Dendropsophus allenorum foi o primeiro para o Brasil. Comparando a eficiência dos métodos de coleta, a busca ativa por coletores capacitados (PLT) foi o método mais eficaz na amostragem da herpetofauna; seguido das AIQ, eficientes na captação de anuros terrícolas e lagartos de serrapilheira nas florestas de terra firme. Não utilizamos AIQ nas áreas de várzea por estas terem solos hidricamente saturados. As AIF, apesar de menos eficientes na amostragem de anuros e lagartos, foram eficazes na captura serpentes e facilmente adaptáveis aos solos hidromórficos das várzeas. Uma maior riqueza de espécies e famílias foi encontrada nas florestas de terra firme. As florestas de várzea tiveram uma marcante ausência de famílias 
fossoriais e de serrapilheira. No entanto, nas florestas de várzea encontramos um maior número de espécies de anuros arborícolas (Hylidae) e de lagartos heliotérmicos maiores (Teiidae). Entretanto, avaliando separadamente cada período de amostragem, a riqueza de espécies da herpetofauna torna-se similar entre os diferentes tipos de paisagens florestais, tendendo ser maior nas florestas de terra firme. Apesar de similares em número espécies, as florestas de terra firme e de várzea foram diferentes quanto ao conjunto das espécies. A diversidade complementar de anfíbios e répteis Squamata entre florestas de terra firme e de várzea (diversidade beta- $\beta$ ), paisagens de maior abrangência local, possivelmente representa a maior contribuição para a diversidade regional da herpetofauna no baixo rio Purus (diversidade Gama- $\gamma$ ).

Palavras-chave: Amazônia brasileira, florestas inundáveis e não-inundáveis, distribuição de espécies, herpetofauna.

\section{Introdução}

Diversas áreas de relevante interesse para conservação de anfíbios e répteis foram identificadas na Amazônia brasileira (Azevedo-Ramos \& Galatti 2001, Brasil 2001, Vogt et al. 2001). Muitas destas áreas de interesse, como a bacia de drenagem do rio Purus, representam lacunas no conhecimento da diversidade destes grupos taxonômicos na região amazônica (Avila-Pires 1995, Azevedo-Ramos \& Gallati 2001, Brasil 2001, Vogt et al. 2001).

Os estudos pioneiros com anfíbios na região do rio Purus foram desenvolvidos por Heyer (1976) que considerou a região em uma análise zoogeográfica dos anfíbios anuros no Bioma Amazônico. O mesmo autor também publicou uma lista descrevendo as espécies de anuros encontradas durante uma expedição onde percorreu o rio Purus desde o estado do Acre até a foz no rio Solimões, estado do Amazonas (Heyer 1977). Posteriormente, poucos estudos inventariaram a diversidade de anfíbios e répteis Squamata nas regiões do médio Purus (Avila-Pires et al. 2009, França \& Venâncio 2010) e do alto rio Purus no Peru (Rodríguez 2003).

O recente processo de implantação de novas unidades de conservação no baixo Purus tem aumentado o conhecimento sobre a diversidade de anfíbios e répteis Squamata na região (Gordo 2003, Waldez \& Vogt 2007, 2009, 2011, Instituto... 2010), principalmente, a partir da criação da Reserva de Desenvolvimento Sustentável Piagaçu-Purus (RDS-PP) (Figura 1) em uma área contígua a Reserva Biológica do Abufarí (REBIO Abufarí) (Albernaz \& Venticinque 2003). A REBIO Abufarí é considerada uma área de alta importância para a conservação de anfíbios e répteis na Amazônia (AzevedoRamos \& Gallati 2001, Brasil 2001, Vogt et al. 2001).

A porção baixa da bacia do rio Purus apresenta uma paisagem formada por um mosaico de áreas de florestas não-inundáveis de terra firme e de florestas sazonalmente inundáveis (Haugaasen \& Peres 2006). As florestas de terra firme correspondem às áreas localizadas acima do nível máximo de inundação dos rios, compondo a maior parte das paisagens florestais da Amazônia com cerca de $80 \%$ da área da bacia (Hess et al. 2003). As florestas inundáveis ocorrem nas planícies de sedimentação dos rios sendo sazonalmente submetidas a um pulso de inundação regular (Junk et al. 1989), alternando entre uma fase aquática e uma fase terrestre (Junk 1997). As florestas de várzea encontram-se associadas aos rios de alta fertilidade e ricos em sedimentos em suspensão (Junk 1997), correspondendo ao segundo maior tipo de paisagem florestal da Amazônia com cerca de 2,6\% da área da bacia (Hess et al. 2003).

Vários estudos têm acessado a diversidade da herpetofauna Amazônica principalmente em áreas de florestas não-inundáveis de terra firme (e.g. Rodríguez \& Duellman 1994, Martins \& Oliveira 1998, Duellman 2005, Bernarde \& Abe 2006, Vogt et al. 2007, Lima et al. 2008, Vitt et al. 2008, Avila-Pires et al. 2009, 2010, Souza 2009, Bernarde et al. 2011). No entanto, poucos trabalhos realizaram análises comparativas entre a diversidade da herpetofauna associada às florestas sazonalmente alagáveis e as florestas de terra firme na
Amazônia (ver Crump 1971, Gascon 1996, Gascon et al. 2000, Doan \& Arizábal 2002, Gordo 2003, Neckel-Oliveira \& Gordo 2004).

A bacia de drenagem do rio Purus apresenta bom estado de conservação com cobertura de floresta primária correspondendo à maior parte da área total (Martins Junior \& Waichman 2009). Entretanto, recentes modelos de desflorestamento futuro na Amazônia prevêem um aumento na perda de cobertura vegetal para região da RDS-PP, relacionado à reativação da rodovia BR-319 (Manaus - Porto Velho) que percorre o interflúvio entre os rios Madeira e Purus (Soares-Filho et al. 2006, Fearnside et al. 2009).

Avaliar comunidades biológicas na região do baixo rio Purus torna-se estratégico para determinar futuros impactos na biodiversidade decorrentes de mudanças na cobertura vegetal. Anfíbios e répteis Squamata são componentes importantes da biomassa e da diversidade de vertebrados em florestas tropicais (e.g. Duellman 1990, Allmon 1991). Ainda, comunidades de anfíbios e pequenos répteis em áreas de floresta amazônica apresentam rápida resposta às alterações na cobertura florestal (e.g. Tocher et al. 1997, Gardner et al. 2007, Bernarde \& Macedo 2008, Souza et al. 2008), mesmo às alterações discretas como no corte florestal seletivo (Vitt et al. 1998).

Visto o parco conhecimento sobre a herpetofauna no baixo rio Purus, o objetivo deste estudo foi inventariar a fauna de anfíbios e répteis Squamata que ocorre associada às florestas na região. Mais especificamente, realizamos uma amostragem ampla em área, utilizando técnicas complementares de coleta da herpetofauna, para comparar a diversidade de espécies associadas às florestas nãoinundáveis de terra firme e as florestas sazonalmente inundáveis de várzea. Também comparamos a eficiência dos métodos de amostragem empregados para acessar a riqueza da herpetofauna nestes dois tipos de florestas.

\section{Material e Métodos}

\section{1. Área de estudo}

As amostragens foram conduzidas na Reserva de Desenvolvimento Sustentável Piagaçu-Purus (RDS-PP, entre $4^{\circ} 03^{\prime}-5^{\circ} 25^{\prime}$ S e $61^{\circ} 40^{\prime}$ $63^{\circ} 30^{\prime} \mathrm{W}$, datum WGS 84) e entorno, região do baixo rio Purus, Amazônia Central, estado do Amazonas - Brasil (Figura 1). Com uma área de 834.245 ha, a RDS-PP abrange um mosaico de grandes paisagens contínuas de florestas de terra firme e florestas inundáveis de várzea e igapó, em ambos interflúvios do rio Purus (Instituto... 2010). O relevo da RDS-PP é homogêneo com altitudes menores que $100 \mathrm{~m}$ a.n.m. (Albernaz \& Venticinque 2003). O clima na região é Equatorial com sub-seca ( $A f$ - classificação Köppen-Geiger) e médias mensais de temperatura acima de $18{ }^{\circ} \mathrm{C}$ e precipitação anual de 2000 a $2600 \mathrm{~mm}$ (Instituto... 2002). De acordo com Silva et al. (2008), a pluviosidade varia sazonalmente, normalmente com a estação chuvosa distribuída entre novembro e março, quando ocorre a alagação da planície de inundação do rio (cheia); e a estação 
Waldez, F. et al.
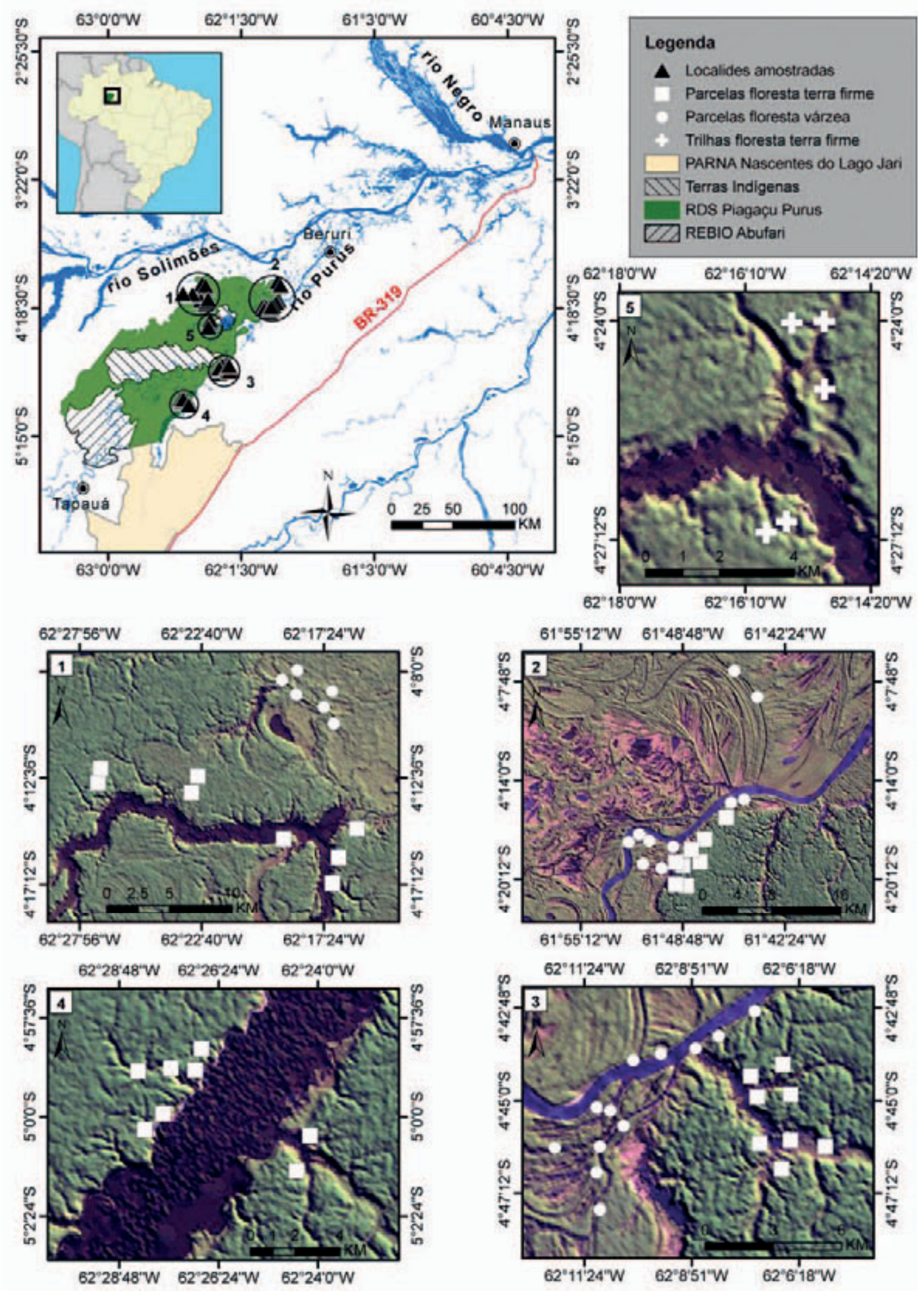

Figura 1. Localização da área de estudos, RDS Piagaçu-Purus, e dos pontos de amostragem, no mosaico regional de áreas protegidas da região do baixo rio Purus na Amazônia Central, Brasil. As imagens de satélite Landsat numeradas de acordo com as sub-regiões indicadas no mapa (figura superior esquerda) mostram as 65 localidades avaliadas, diferenciadas por estratégias de amostragem e por tipo de habitat florestal.

Figure 1. Geographical location of the study area, Piagaçu-Purus Reserve, and sampling plots, in the regional mosaic of protected areas from the lower Purus River Basin, Central Amazonia, Brazil. The Landsat satellite images were numbered according to the sub-regions indicated in the map (upper left figure) and indicate the 65 localities evaluated and differentiated by the sampling strategies and types of forest habitats. 
de estiagem distribuída entre maio e setembro, quando ocorre a drenagem da planície de inundação do rio (seca), com abril e outubro correspondendo à períodos de transição entre estações. O nível de água no baixo rio Purus pode variar até $12 \mathrm{~m}$ entre estações, sendo influenciado tanto pela quantidade de chuvas, quanto por flutuações do médio rio Solimões (Goulding et al. 2003).

As áreas estudadas corresponderam às paisagens de florestas de terra firme e de várzea (Figura 2a, b). As florestas de terra firme ocupam $50 \%$ da área da RDS-PP e ocorrem nas maiores altitudes ( $\geq 40$ m a.n.m.), acima da planície de inundação do rio (Instituto... 2010). Estas florestas têm sub-bosque denso e alta diversidade de árvores, com Lecythidaceae e Chrysobalanaceae como principais famílias arbóreas (Haugaasen \& Peres 2006). Nesta região são encontradas manchas de floresta denominadas castanhais, com altas densidades de árvores de castanha-da-Amazônia (Bertholletia excelsa Humboldt \& Bonpland - Lecythidaceae) e tradicionalmente utilizadas pelas populações locais como fontes de sementes comestíveis (Instituto... 2010, Haugaasen \& Peres 2006).

As florestas de várzea ocupam $45 \%$ da área da RDS-PP e ocorrem em altitudes menores que $40 \mathrm{~m}$ a.n.m. sendo sazonalmente alagado com a inundação da planície pelo rio Purus (Instituto... 2010). Estas florestas possuem sub-boque mais aberto e menor diversidade relativa de árvores, com Euphorbiaceae e leguminosas (Caesalpiniaceae, Fabaceae e Mimosaceae) como principais famílias arbóreas (Haugaasen \& Peres 2006).

\section{Estratégias de amostragem}

O estudo da herpetofauna nestas paisagens foi realizado em dois diferentes períodos, ambos com distintas estratégias de amostragem. O primeiro período ocorreu no final de seca do rio de 2004 (outubro a dezembro - 60 dias) e durante o máximo de cheia do rio de 2005 (fevereiro a maio - 60 dias). Neste período, a amostragem foi conduzida na cabeceira do Lago Ayapuá, com cinco transectos de $1 \mathrm{~km}$ dispostos perpendicularmente aos corpos d'água (Figura 1, Quadro 5). Cada trilha atravessou uma área maior de floresta de terra firme e uma curta faixa de floresta de várzea $(<100 \mathrm{~m})$ entre o corpo d'água e o início da floresta não-inundável.

Em cada um dos transectos foram instaladas armadilhas de interceptação e queda - AIQ (pitfall traps with drift fence) (Cechin \& Martins 2000) dispostas em linha, com cada conjunto composto
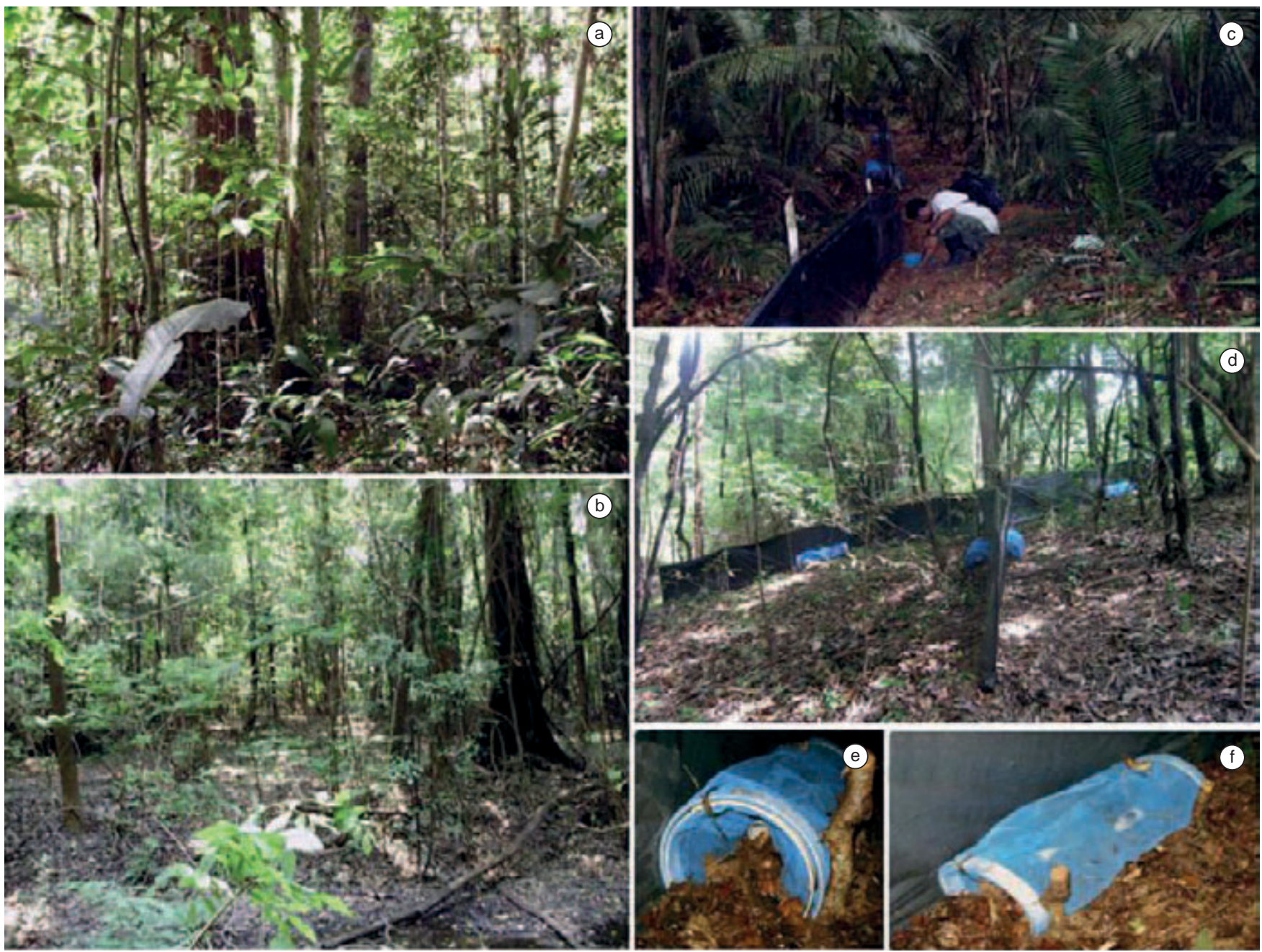

Figura 2. Florestas de terra firme (a) e florestas de várzea (b) na região do baixo rio Purus, Amazônia Central - Brasil. Estas paisagens foram pesquisadas quanto à diversidade de anfíbios e répteis Squamata com o uso de armadilhas de interceptação e queda com funis duplos em linhas (c) e armadilhas de interceptação com funis duplos em $\mathrm{Y}(\mathrm{d}, \mathrm{e}, \mathrm{f})$.

Figure 2. The terra firme nonflooded forests (a) and the varzea flooded forests (b) from the lower Purus River Basin, Central Amazonia, Brazil. These landscapes were searched for the diversity of amphibians and Squamata reptiles, and we used technical sampling of lines of pitfall traps with drift fence interpolated with double-ended funnel traps (c) and Y-lines of double-ended funnel traps with drift fence (d, e, f). 
por 10 baldes de $60 \mathrm{~L}$ ligados por $10 \mathrm{~m}$ de cerca plástica com $1 \mathrm{~m}$ de altura enterrada no solo (esforço de AIQ $=144.000$ horas com 50 baldes) (Figura 2c). Funis duplos (double-ended funnel traps) (Greenberg et al. 1994) foram adicionados aos conjuntos de AIQ. Essas armadilhas de interceptação com funis duplos - AIF utilizaram funis com aberturas circulares de $5 \mathrm{~cm}$ de diâmetro e $1 \mathrm{~m}$ de comprimento, dispostos entre os baldes nos dois lados da cerca. Um total de 18 funis foi adicionado a cada conjunto de AIQ (esforço de $\mathrm{AIF}=259.200$ horas com 90 funis) (Figura 2c, e, f). Nestas trilhas dois observadores registraram espécies ativamente em períodos diurno e noturno (procura limitada por tempo - PLT $=252.6$ horas) por meio de encontro visual (Crump \& Scott 1994).

O segundo período de amostragem ocorreu durante a cheia do rio em 2009 (maio a julho - 60 dias) e em 2010 (fevereiro - 20 dias, maio a julho - 60 dias), e durante a seca do rio em 2009 (outubro a dezembro - 60 dias) e em 2010 (outubro a dezembro - 60 dias). Neste segundo período, as espécies foram amostradas em 60 parcelas distribuídas em florestas de terra firme ( 32 parcelas) e florestas de várzeas (28 parcelas) que abrangeram paisagens nos dois interflúvios do baixo rio Purus (Figura 1, quadros 1, 2, 3 e 4). Em cada uma dessas localidades, foram instaladas parcelas de tamanho $10 \times 250 \mathrm{~m}$ (0,25 ha). Outros estudos com herpetofauna na Amazônia brasileira têm utilizado parcelas de tamanhos similares (Menin et al. 2007, 2008, 2011, Rojas-Ahumada \& Menin 2010, Fraga et al. 2011).

Nestas parcelas, as espécies foram buscadas ativamente por um mínimo de dois e um máximo de quatro pessoas. Cada observador registrou as espécies distanciando-se até $5 \mathrm{~m}$ do centro da parcela. Cada parcela foi amostrada duas vezes em período diurno e duas vezes em período noturno, com um intervalo máximo de oito dias entre cada amostragem (procura limitada por tempo - PLT $=850,9$ horas). Nestas ocasiões, as parcelas foram lentamente percorridas e as espécies procuradas no chão e na vegetação, sendo registradas por encontro visual (Crump \& Scott 1994) e por amostragem auditiva (Zimmerman 1994). Em cada parcela também foram instaladas armadilhas de interceptação com funis duplos - AIF (double-ended funnel traps with drift fence) (Greenberg et al. 1994), contendo seis funis duplos ligados por uma cerca plástica guia em forma de $\mathrm{Y}$, cada braço com $6 \mathrm{~m}$ de comprimento, $1 \mathrm{~m}$ de altura e enterrado no solo (esforço de $\mathrm{AIF}=38.304$ horas) (Figura 2d, e, f).

Para maximizar a independência de amostragem entre as localidades estudadas, todas as unidades amostrais (transectos e parcelas) foram distribuídas com uma distância mínima de 1 km entre as mesmas. O primeiro autor participou de todas as coletas e as espécies observadas fora das unidades amostrais foram contabilizadas como registros ocasionais. Espécimes testemunhos foram coletados (licenças de coleta IBAMA 02005.001919/0098 \& 20059-1) e depositados na coleção de Anfíbios e Répteis do Instituto Nacional de Pesquisas da Amazônia (INPA) em Manaus, Amazonas - Brasil.

\section{Análise de dados}

A riqueza de anfíbios, lagartos e serpentes foi avaliada graficamente e por meio de curvas de rarefação, construídas por localidade amostrada e agrupando os dados para cada tipo de floresta. Essas curvas consideraram o número total de indivíduos em intervalos de confiança de $95 \%$, a fim de padronizar o efeito dos diferentes métodos de coleta e estratégias de amostragem (Gotelli \& Colwell 2001, Colwell 2004), utilizados em cada tipo de habitat nos dois períodos de estudo.

As curvas de rarefação foram graficamente comparadas com curvas de estimativa da riqueza local para cada grupo (sapos, lagartos e serpentes), também construídas por localidade e agrupando dados para cada tipo de floresta. Estas curvas de estimativa de riqueza representaram o valor médio obtido a partir de estimativas calculadas utilizando os quatro estimadores de riqueza baseados em abundância mais correntemente empregados (ACE, Chao 1, Jack 1 e Bootstrap) (ver Colwell 2004). O uso da média desses estimadores de riqueza minimiza variações no desempenho particular de estimadores, normalmente relacionadas às diferenças em diversidade, amostragem e equitabilidade das comunidades (O'Hara 2005). Todas as curvas de rarefação e de estimativa da riqueza local foram obtidas com o programa EstimateS v.7 (Colwell 2004), para tanto não contabilizamos os registros eventuais das espécies.

\section{Resultados}

Um total de 160 espécies e 5.561 indivíduos foi registrado neste estudo, entre os quais, espécies de anfíbios (Figura 3) e de répteis Squamata (Figura 4) pouco conhecidas para a região do baixo rio Purus. Deste total de espécies, 75 taxa foram anfíbios representados por 11 famílias de anuros e duas famílias de gimnofionas (Tabela 1); e 85 taxa foram répteis Squamata representados por nove famílias de lagartos e oito famílias de serpentes (Tabela 2).

A diversidade observada neste estudo foi próxima da riqueza local estimada para anfíbios e lagartos, tanto nas florestas de várzea quanto nas florestas de terra firme, mas abaixo da riqueza local esperada para serpentes em ambos os tipos de florestas (Figura 5).

Para todas as taxocenoses avaliadas foi observada maior riqueza de espécies nas florestas de terra firme que nas florestas de várzea: anfíbios (60/43 spp.), lagartos (31/19 spp.) e serpentes (41/27 spp.) (Figura 6). No entanto, as florestas de várzea abrigaram maior riqueza de lagartos heliotérmicos de maior porte (Teiidae) e de sapos arborícolas (Hylidae).

No geral, um maior esforço de amostragem foi empregado nas florestas de terra firme que nas florestas de várzea (ver Estratégias de amostragem). Este desbalanço em esforço foi evidenciado pelo maior número de espécimes encontrados nas florestas de terra firme, mais que o triplo dos registros feitos nas florestas de várzea: anfíbios (2566/763 espécimes), lagartos (1536/450 espécimes) e serpentes (116/36 espécimes).

No entanto, quando comparamos a riqueza das taxocenoses avaliadas nos ambientes, entre os diferentes períodos de amostragem, um número de espécies mais similar foi evidenciado entre os tipos de paisagens florestais, para os períodos que empregaram esforço de coleta e estratégias de amostragem mais similares (Tabela 3). Também, foram expostas as variações de eficiência para cada método de amostragem empregado (ver Tabela 3). A busca ativa limitada por tempo (PLT) foi o método que no geral representou uma maior riqueza de espécies para todas as taxocenoses avaliadas nos dois tipos de florestas. Seguida das armadilhas de interceptação e queda (AIQ), que representaram uma maior riqueza de sapos terrícolas e principalmente de lagartos de serrapilheira nas florestas de terra firme. Finalmente, das armadilhas de interceptação com funis de dupla entrada (AIF), ineficientes na captura dos taxa melhores amostrados pelas AIQ, mas com melhor desempenho na amostragem da riqueza de serpentes terrícolas.

\section{Discussão}

A riqueza de espécies de anfíbios e répteis Squamata na região do baixo rio Purus foi similar a diversidade encontrada em outras áreas de floresta na planície Amazônica (e.g. Rodríguez \& Duellman 1994, Martins \& Oliveira 1998, Donnelly et al. 2005, Duellman 1999, 2005, Bernarde \& Abe 2006, Vogt et al. 2007, Avila-Pires et al. 2009, 2010, Lima et al. 2008, Vitt et al. 2008), em particular, com localidades na mesma área de endemismo (ver Rodríguez 2003, Souza 2009, França \& Venâncio 2010, Instituto... 2010, Bernarde et al. 2011). 


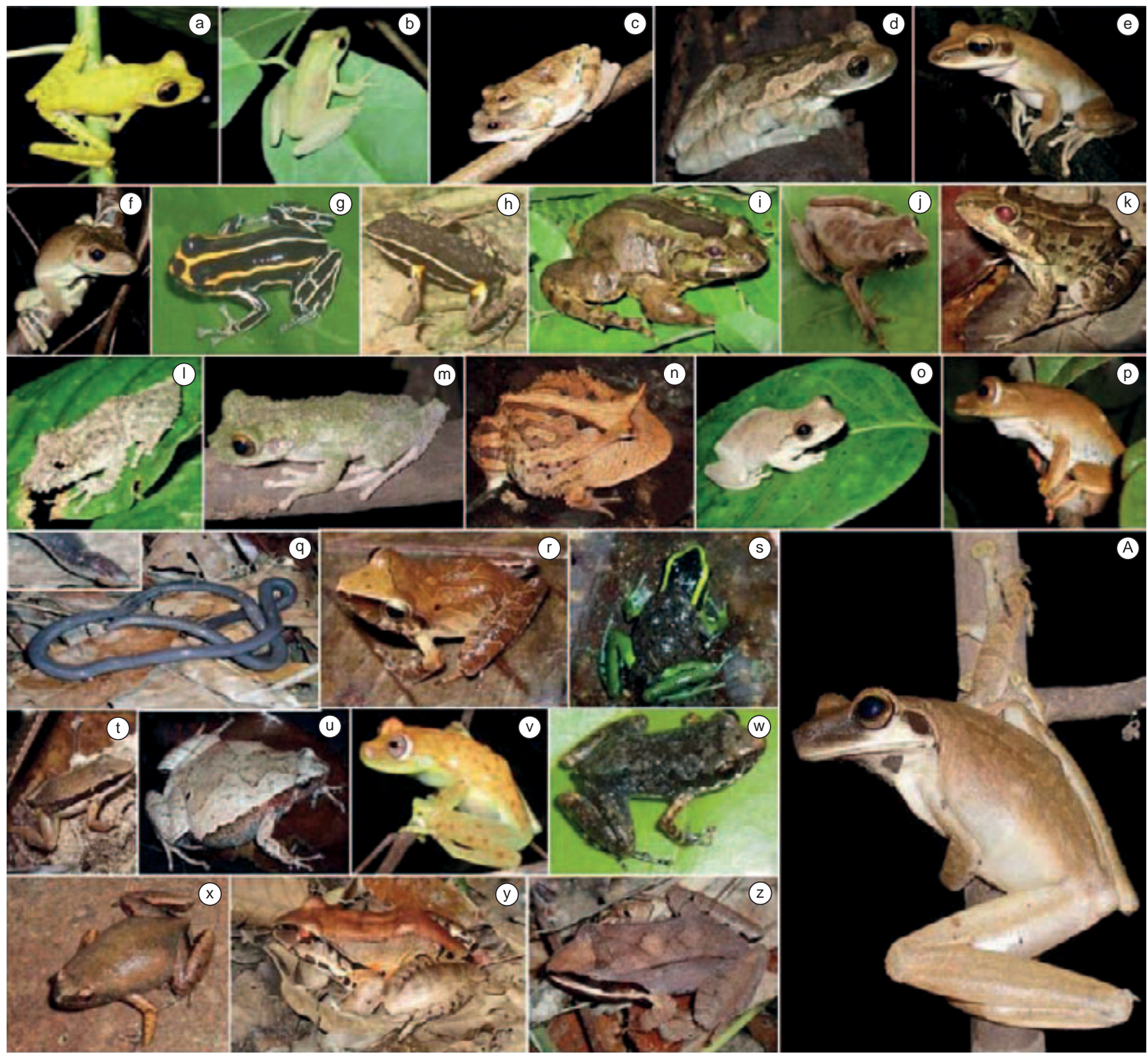

Figura 3. Espécies de anfíbios observadas na região do baixo rio Purus, Amazônia Central, Brasil: a) Hypsiboas fasciatus; b) Scarthyla goinorum; c) Dendropsophus allenorum; d) Trachycephalus typhonius; e) Hypsiboas lanciformis; f) Hypsiboas wavrini; g) Ranitomeya ventrimaculata; h) Ameerega hahneli; i) Hydrolaetare schmidti; j) Dendropsophus koechlini; k) Leptodactylus macrosternum; 1) Scinax pedromedinae; m) Osteocephalus cf. buckleyi; n) Ceratophrys cornuta; o) Scinax boesemani; p) Hypsiboas geographicus; q) Oscaecilia aff. bassleri; r) Pristimantis conspicillatus; s) Ameerega trivittata; t) Allobates sp.2; u) Hamptophryne boliviana; v) Hypsiboas punctatus; w) Phyzelaphryne miriamae; x) Chiasmocleis avilapiresae; y) Leptodactylus pentadactylus; z) Leptodactylus mystaceus e A) Osteocephalus planiceps (Fotos - F. Waldez).

Figure 3. Species of amphibians observed in the lower Purus River Basin, Central Amazonia, Brazil: a) Hypsiboas fasciatus; b) Scarthyla goinorum; c) Dendropsophus allenorum; d) Trachycephalus typhonius; e) Hypsiboas lanciformis; f) Hypsiboas wavrini; g) Ranitomeya ventrimaculata; h) Ameerega hahneli; i) Hydrolaetare schmidti; j) Dendropsophus koechlini; k) Leptodactylus macrosternum; 1) Scinax pedromedinae; m) Osteocephalus cf. buckleyi; n) Ceratophrys cornuta; o) Scinax boesemani; p) Hypsiboas geographicus; q) Oscaecilia aff. bassleri; r) Pristimantis conspicillatus; s) Ameerega trivittata; t) Allobates sp.2; u) Hamptophryne boliviana; v) Hypsiboas punctatus; w) Phyzelaphryne miriamae; x) Chiasmocleis avilapiresae; y) Leptodactylus pentadactylus; z) Leptodactylus mystaceus and A) Osteocephalus planiceps (Photos - F. Waldez).

A diversidade de vertebrados na Amazônia tem sido relacionada à ocorrência de áreas de endemismo (Silva et al. 2005) e padrões de distribuição coincidentes foram evidenciados para espécies de sapos (Duellman 1999, Ron 2000) e lagartos (Avila-Pires 1995, AvilaPires et al. 2009). A bacia de drenagem do rio Purus localiza-se na área de endemismo amazônica denominada Inambari sensu Cracraft
(1985). Esta região biogeográfica corresponde à planície cisandina delimitada desde a confluência dos rios Amazonas/Solimões com o rio Negro no Brasil, seguindo a oeste pela margem sul do Amazonas/ Solimões através do Brasil e Peru e a leste pela margem oeste do rio Madeira através do Brasil, Bolívia e Peru (ver Silva et al. 2005). Uma análise preliminar da herpetofauna do baixo rio Purus comparou 
Waldez, F. et al.

Tabela 1. Espécies de anfíbios encontradas na região do baixo rio Purus, Amazônia Central, Brasil. O número de espécimes foi indicado por: tipo de ambiente (floresta de terra firme e floresta de várzea), tipo de unidade amostral (parcela e transectos) e métodos de coleta (armadilha de interceptação com funis duplos - AIF, armadilha de interceptação e queda- AIQ, procura limitada por tempo - PLT e registro ocasional).

Table 1. Species of amphibians found in the lower Purus River Basin, Central Amazonia, Brazil. The individual numbers are indicated by: type of forest habitat (terra firmenonflooded forests and várzea flooded forests), sampling unit (plot and transect) and sampling method (time constrained audio-visual search - PLT, pitfall traps with drift fence-AIQ, double-ended funnel traps with drift fence-AIF and occasional records).

\begin{tabular}{|c|c|c|c|c|c|c|c|c|c|}
\hline \multirow[t]{2}{*}{ AMPHIBIA : LISSAMPHIBIA (75 spp.) } & \multirow[t]{2}{*}{ Total } & \multicolumn{2}{|c|}{$\begin{array}{c}\text { Parcelas } \\
\text { várzea }(\mathrm{N}=28) \\
\end{array}$} & \multicolumn{2}{|c|}{$\begin{array}{l}\text { Parcelas terra } \\
\text { firme }(\mathrm{N}=32)\end{array}$} & \multicolumn{3}{|c|}{$\begin{array}{c}\text { Transectos terra } \\
\text { firme }(\mathrm{N}=5)\end{array}$} & \multirow{2}{*}{$\begin{array}{l}\text { Registros } \\
\text { ocasionais }\end{array}$} \\
\hline & & AIF & PLT & AIF & PLT & AIQ & AIF & PLT & \\
\hline Ordem ANURA (73 spp.) & 3384 & 14 & 749 & 96 & 1249 & 561 & 148 & 511 & 57 \\
\hline \multicolumn{10}{|l|}{ Aromobatidae (3 spp.) } \\
\hline Allobates femoralis (Boulenger, 1884 “1883”) & 67 & 0 & 10 & 4 & 13 & 27 & 1 & 12 & 0 \\
\hline Allobates sp.1 & 8 & 0 & 0 & 0 & 8 & 0 & 0 & 0 & 0 \\
\hline Allobates sp.2 & 22 & 0 & 0 & 0 & 14 & 6 & 0 & 2 & 0 \\
\hline \multicolumn{10}{|l|}{ Bufonidae (4 spp.) } \\
\hline Amazophrynella aff.bokermanni (Izecksohn, 1994 “1993”) & 2 & 0 & 0 & 0 & 2 & 0 & 0 & 0 & 0 \\
\hline Rhinella margaritifera (Laurenti, 1768) & 318 & 1 & 4 & 14 & 152 & 58 & 21 & 68 & 0 \\
\hline Rhinella marina (Linnaeus, 1758) & 98 & 5 & 23 & 0 & 52 & 9 & 1 & 8 & 0 \\
\hline Rhinella merianae Gallardo, 1965 & 36 & 0 & 0 & 0 & 1 & 0 & 0 & 20 & 15 \\
\hline \multicolumn{10}{|l|}{ Ceratophryidae (1 sp.) } \\
\hline Ceratophrys cornuta (Linnaeus, 1758) & 1 & 0 & 0 & 0 & 0 & 1 & 0 & 0 & 0 \\
\hline \multicolumn{10}{|l|}{ Dendrobatidae (4 spp.) } \\
\hline Adelphobates quinquevittatus (Steindachner, 1864) & 3 & 0 & 0 & 0 & 2 & 1 & 0 & 0 & 0 \\
\hline Ameerega hahneli (Boulenger, 1884 “1883”) & 205 & 0 & 0 & 10 & 124 & 18 & 35 & 18 & 0 \\
\hline Ameerega trivittata (Spix, 1824) & 37 & 0 & 0 & 0 & 14 & 9 & 0 & 14 & 0 \\
\hline Ranitomeya ventrimaculata (Shreve, 1935) & 2 & 0 & 0 & 0 & 1 & 0 & 0 & 1 & 0 \\
\hline \multicolumn{10}{|l|}{ Eleutherodactylidae (1 sp.) } \\
\hline Phyzelaphryne miriamae Heyer, 1977 & 15 & 0 & 0 & 0 & 10 & 5 & 0 & 0 & 0 \\
\hline \multicolumn{10}{|l|}{ Hylidae (33 spp.) } \\
\hline Dendropsophus allenorum (Duellman and Trueb, 1989) & 46 & 0 & 44 & 0 & 2 & 0 & 0 & 0 & 0 \\
\hline Dendropsophus brevifrons (Duellman \& Crump, 1974) & 2 & 0 & 2 & 0 & 0 & 0 & 0 & 0 & 0 \\
\hline Dendropsophus haraldschultzi (Bokermann, 1962) & 2 & 0 & 1 & 0 & 0 & 0 & 0 & 0 & 1 \\
\hline Dendropsophus koechlini (Duellman \& Trueb, 1989) & 1 & 0 & 1 & 0 & 0 & 0 & 0 & 0 & 0 \\
\hline Dendropsophus leali (Bokermann, 1964) & 41 & 0 & 20 & 0 & 0 & 0 & 0 & 21 & 0 \\
\hline Dendropsophus leucophyllatus (Beireis, 1783) & 191 & 0 & 17 & 0 & 127 & 0 & 0 & 47 & 0 \\
\hline Dendropsophus rossalleni (Goin, 1959) & 6 & 0 & 0 & 0 & 1 & 0 & 0 & 5 & 0 \\
\hline Dendropsophus triangulum (Günther, 1869 “1868”) & 3 & 0 & 1 & 0 & 0 & 0 & 0 & 0 & 2 \\
\hline Hypsiboas calcaratus (Troschel in Schomburgk, 1848) & 25 & 0 & 12 & 0 & 4 & 0 & 0 & 9 & 0 \\
\hline Hypsiboas cinerascens (Spix, 1824) & 2 & 0 & 1 & 0 & 1 & 0 & 0 & 0 & 0 \\
\hline Hypsiboas fasciatus (Günther, 1859 “1858”) & 56 & 0 & 43 & 0 & 13 & 0 & 0 & 0 & 0 \\
\hline Hypsiboas geographicus (Spix, 1824) & 37 & 0 & 31 & 0 & 1 & 0 & 0 & 5 & 0 \\
\hline Hypsiboas lanciformis (Cope, 1871) & 12 & 0 & 4 & 0 & 6 & 0 & 0 & 2 & 0 \\
\hline Hypsiboas punctatus (Schneider, 1799) & 4 & 0 & 3 & 0 & 1 & 0 & 0 & 0 & 0 \\
\hline Hypsiboas raniceps Cope, 1862 & 18 & 0 & 5 & 0 & 0 & 0 & 0 & 8 & 5 \\
\hline Hypsiboas wavrini (Parker, 1936) & 71 & 0 & 22 & 0 & 12 & 0 & 0 & 37 & 0 \\
\hline Osteocephalus cf. buckleyi (Boulenger, 1882) & 2 & 0 & 1 & 0 & 1 & 0 & 0 & 0 & 0 \\
\hline Osteocephalus cabrerai (Cochran \& Goin, 1970) & 1 & 0 & 0 & 0 & 1 & 0 & 0 & 0 & 0 \\
\hline Osteocephalus leprieurii (Duméril \& Bibron, 1841) & 4 & 0 & 0 & 0 & 4 & 0 & 0 & 0 & 0 \\
\hline Osteocephalus planiceps Cope, 1874 & 43 & 0 & 16 & 0 & 27 & 0 & 0 & 0 & 0 \\
\hline Osteocephalus taurinus Steindachner, 1862 & 153 & 0 & 58 & 0 & 50 & 0 & 0 & 45 & 0 \\
\hline Phyllomedusa vaillantii Boulenger, 1882 & 3 & 0 & 0 & 0 & 3 & 0 & 0 & 0 & 0 \\
\hline Pseudis limellum (Cope, 1862) & 41 & 0 & 29 & 0 & 0 & 0 & 0 & 0 & 12 \\
\hline Scarthyla goinorum (Bokermann, 1962) & 44 & 0 & 42 & 1 & 1 & 0 & 0 & 0 & 0 \\
\hline Scinax boesemani (Goin, 1966) & 39 & 0 & 26 & 0 & 3 & 0 & 0 & 10 & 0 \\
\hline Scinax garbei (Miranda-Ribeiro, 1926) & 109 & 0 & 47 & 0 & 37 & 0 & 0 & 25 & 0 \\
\hline Scinax nebulosus (Spix, 1824) & 5 & 0 & 1 & 0 & 1 & 0 & 0 & 3 & 0 \\
\hline Scinax pedromedinae (Henle, 1991) & 33 & 0 & 33 & 0 & 0 & 0 & 0 & 0 & 0 \\
\hline
\end{tabular}


Tabela 1. Continuação...

\begin{tabular}{|c|c|c|c|c|c|c|c|c|c|}
\hline \multirow[t]{2}{*}{ AMPHIBIA : LISSAMPHIBIA (75 spp.) } & \multirow[t]{2}{*}{ Total } & \multicolumn{2}{|c|}{$\begin{array}{c}\text { Parcelas } \\
\text { várzea }(N=28)\end{array}$} & \multicolumn{2}{|c|}{$\begin{array}{l}\text { Parcelas terra } \\
\text { firme }(\mathrm{N}=32)\end{array}$} & \multicolumn{3}{|c|}{$\begin{array}{l}\text { Transectos terra } \\
\text { firme }(\mathbf{N}=\mathbf{5})\end{array}$} & \multirow{2}{*}{$\begin{array}{l}\text { Registros } \\
\text { ocasionais }\end{array}$} \\
\hline & & AIF & PLT & AIF & PLT & AIQ & AIF & PLT & \\
\hline Scinax ruber (Laurenti, 1768) & 61 & 0 & 39 & 0 & 6 & 0 & 0 & 16 & 0 \\
\hline Sphaenorhynchus carneus (Cope, 1868) & 2 & 0 & 0 & 0 & 0 & 0 & 0 & 0 & 2 \\
\hline Sphaenorhynchus lacteus (Daudin, 1800) & 2 & 0 & 0 & 0 & 0 & 0 & 0 & 0 & 2 \\
\hline Trachycephalus resinifictrix (Goeldi, 1907) & 127 & 0 & 55 & 0 & 43 & 0 & 0 & 28 & 1 \\
\hline Trachycephalus typhonius (Linnaeus, 1758) & 23 & 0 & 16 & 0 & 0 & 0 & 0 & 7 & 0 \\
\hline \multicolumn{10}{|l|}{ Leiuperidae (1 sp.) } \\
\hline Engystomops freibergi (Donoso-Barros, 1969) & 32 & 0 & 0 & 0 & 0 & 27 & 5 & 0 & 0 \\
\hline \multicolumn{10}{|l|}{ Leptodactylidae (11 spp.) } \\
\hline Hydrolaetare schmidti (Cochran \& Goin, 1959) & 2 & 0 & 0 & 0 & 2 & 0 & 0 & 0 & 0 \\
\hline Leptodactylus aff. andreae Müller, 1923 & 860 & 3 & 98 & 65 & 382 & 229 & 23 & 60 & 0 \\
\hline Leptodactylus fuscus (Schneider, 1799) & 20 & 0 & 0 & 0 & 0 & 0 & 0 & 8 & 12 \\
\hline Leptodactylus knudseni Heyer, 1972 & 10 & 0 & 0 & 0 & 0 & 7 & 3 & 0 & 0 \\
\hline Leptodactylus lineatus (Schneider, 1799) & 31 & 0 & 0 & 0 & 0 & 26 & 4 & 1 & 0 \\
\hline Leptodactylus macrosternum Miranda-Ribeiro, 1926 & 4 & 0 & 4 & 0 & 0 & 0 & 0 & 0 & 0 \\
\hline Leptodactylus mystaceus (Spix, 1824) & 68 & 0 & 0 & 0 & 1 & 41 & 22 & 4 & 0 \\
\hline Leptodactylus pentadactylus (Laurenti, 1768) & 95 & 0 & 16 & 0 & 56 & 6 & 9 & 8 & 0 \\
\hline Leptodactylus petersii (Steindachner, 1864) & 110 & 5 & 20 & 2 & 41 & 29 & 5 & 8 & 0 \\
\hline Leptodactylus rhodomystax Boulenger, 1884 “1883” & 15 & 0 & 0 & 0 & 1 & 4 & 7 & 2 & 1 \\
\hline Leptodactylus stenodema Jiménez de la Espada, 1875 & 9 & 0 & 1 & 0 & 1 & 6 & 1 & 0 & 0 \\
\hline \multicolumn{10}{|l|}{ Microhylidae (7 spp.) } \\
\hline Chiasmocleis avilapiresae Peloso \& Sturaro, 2008 & 35 & 0 & 0 & 0 & 0 & 26 & 9 & 0 & 0 \\
\hline Chiasmocleis bassleri Dunn, 1949 & 27 & 0 & 0 & 0 & 3 & 13 & 2 & 9 & 0 \\
\hline Chiasmocleis hudsoni Parker, 1940 & 1 & 0 & 0 & 0 & 0 & 1 & 0 & 0 & 0 \\
\hline Ctenophryne geayi Mocquard, 1904 & 2 & 0 & 0 & 0 & 0 & 2 & 0 & 0 & 0 \\
\hline Hamptophryne boliviana (Parker, 1927) & 2 & 0 & 0 & 0 & 0 & 2 & 0 & 0 & 0 \\
\hline Synapturanus aff. salseri Pyburn, 1975 & 16 & 0 & 3 & 0 & 13 & 0 & 0 & 0 & 0 \\
\hline Syncope aff. antenori Walker, 1973 & 1 & 0 & 0 & 0 & 0 & 1 & 0 & 0 & 0 \\
\hline \multicolumn{10}{|l|}{ Pipidae (2 spp.) } \\
\hline Pipa pipa (Linnaeus, 1758) & 3 & 0 & 0 & 0 & 1 & 0 & 0 & 0 & 2 \\
\hline Pipa snethlageae Müller, 1914 & 2 & 0 & 0 & 0 & 0 & 0 & 0 & 0 & 2 \\
\hline \multicolumn{10}{|l|}{ Strabomantidae (5 spp.) } \\
\hline Oreobates quixensis Jiménez de la Espada, 1872 & 1 & 0 & 0 & 0 & 1 & 0 & 0 & 0 & 0 \\
\hline Pristimantis altamazonicus (Barbour \& Dunn, 1921) & 4 & 0 & 0 & 0 & 0 & 4 & 0 & 0 & 0 \\
\hline Pristimantis conspicillatus (Günther, 1858) & 8 & 0 & 0 & 0 & 8 & 0 & 0 & 0 & 0 \\
\hline Pristimantis aff. ventrimarmoratus (Boulenger, 1912) & 3 & 0 & 0 & 0 & 0 & 3 & 0 & 0 & 0 \\
\hline Pristimantis sp. & 1 & 0 & 0 & 0 & 1 & 0 & 0 & 0 & 0 \\
\hline Ordem GYMNOPHIONA (2 spp.) & 4 & $\mathbf{0}$ & $\mathbf{0}$ & $\mathbf{0}$ & $\mathbf{0}$ & 1 & $\mathbf{0}$ & $\mathbf{0}$ & 3 \\
\hline \multicolumn{10}{|l|}{ Caeciliidae (1 sp.) } \\
\hline Oscaecilia aff. bassleri (Dunn, 1942) & 1 & 0 & 0 & 0 & 0 & 1 & 0 & 0 & 0 \\
\hline \multicolumn{10}{|l|}{ Typhlonectidae (1 sp.) } \\
\hline Typhlonectes compressicauda (Duméril \& Bibron, 1841) & 3 & 0 & 0 & 0 & 0 & 0 & 0 & 0 & 3 \\
\hline
\end{tabular}

o Coeficiente de Similaridade Biogeográfica (sensu Duellman 1990) com outras localidades na Amazônia e encontrou maior correspondência biogeográfica com áreas na Amazônia Central, no Sudoeste e Oeste da Amazônia (Instituto... 2010), regiões abrangidas pela área de endemismo Inambari (Silva et al. 2005). Este padrão de distribuição foi corroborado pela ocorrência de espécies como: os sapos Dendropsophus allenorum, Pristimantis altamazonicus, Scinax pedromedinae característicos do alto Amazonas/Solimões e sudoeste da Amazônia (Rodríguez \& Duellman 1994, Duellman 1999, Ron
2000, Frost 2011), e os lagartos Gonatodes hasemani, Leposoma osvaldoi, encontrados no sudoeste da Amazônia, e Anolis tandai, Leposoma snethlageae, Uracentron azureum guentheri, encontrados no oeste da Amazônia (Avila-Pires 1995, Avila-Pires et al. 2009).

Nesse estudo, realizamos uma amostragem ampla em esforço e área, utilizando técnicas de coleta complementares eficientes na captação da diversidade da herpetofauna em florestas tropicais (ver Doan 2003, Ribeiro-Júnior et al. 2008). No entanto, para conhecer a diversidade das comunidades de anfíbios e pequenos répteis em 

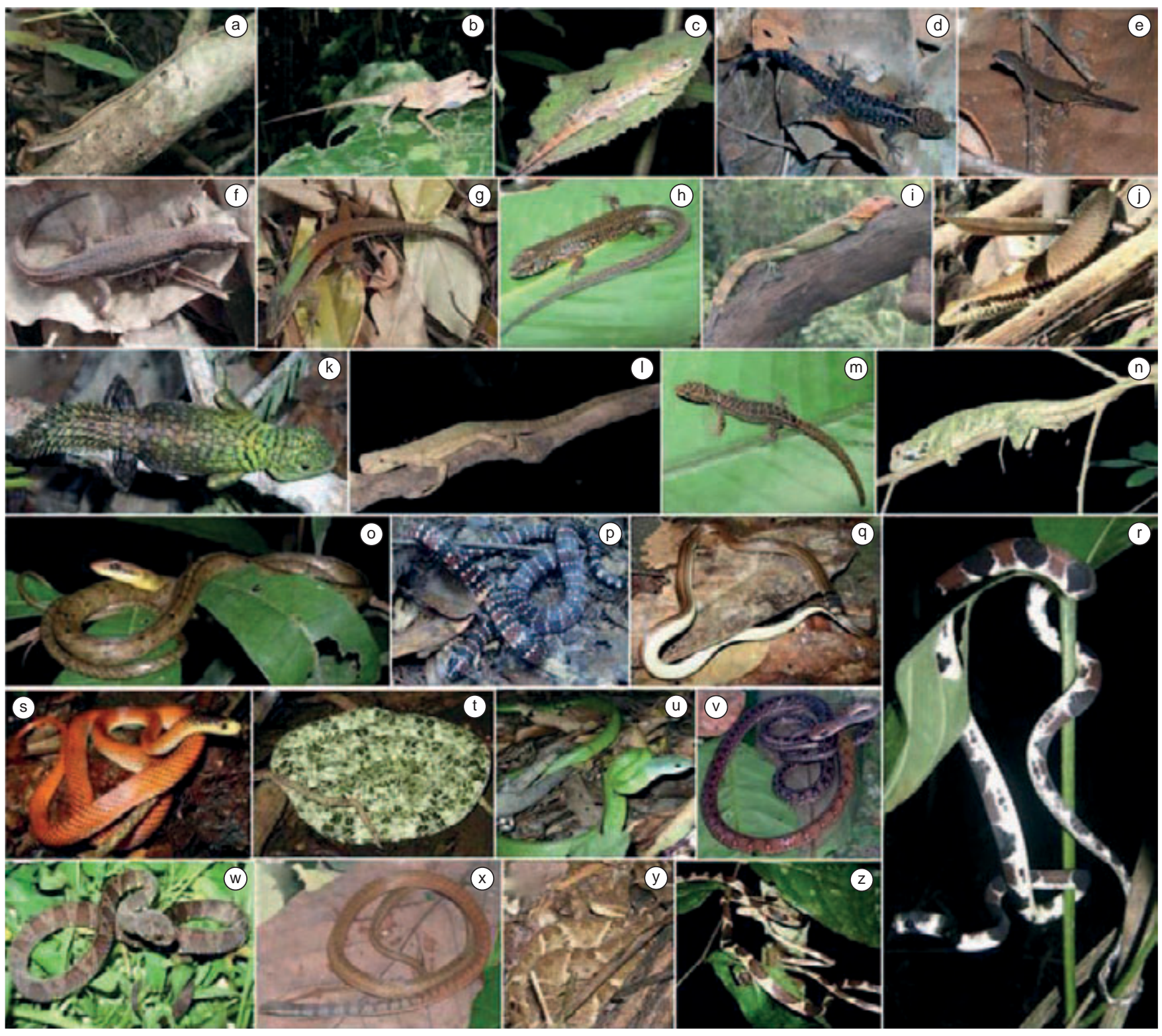

Figura 4. Espécies de répteis Squamata observadas na região do baixo rio Purus, Amazônia Central, Brasil: a) Anolis fuscoauratus; b) Anolis tandai; c) Gonatodes humeralis; d) Gonatodes hasemani; e) Chatogekko amazonicus; f) Alopoglossus aff. atriventris; g) Kentropyx altamazonica; h) Arthrosaura reticulata; i) Dracaena guianensis; j) Copeoglossum nigropunctatum; k) Uracentron azureum guentheri; 1) Uranoscodon superciliosus; m) Lepidoblepharis heyerorum; n) Plica umbra; o) Chironius scurrulus; p) Micrurus langsdorffii; q) Tantilla melanocephala; r) Dipsas catesbyi; s) Oxyrhopus occipitalis; t) Bothriopsis taeniata; u) Liophis typhlus; v) Dendrophidion dendrophis; w) Dipsas indica; x) Taeniophallus aff. occipitalis; y) Bothrops atrox; z) Imantodes cenchoa (Foto i - B. Marioni, demais fotos - F. Waldez).

Figure 4. Species of Squamata reptilians observed in the lower Purus River Basin, Central Amazonia, Brazil: a) Anolis fuscoauratus; b) Anolis tandai; c) Gonatodes humeralis; d) Gonatodes hasemani; e) Chatogekko amazonicus; f) Alopoglossus aff. atriventris; g) Kentropyx altamazonica; h) Arthrosaura reticulata; i) Dracaena guianensis; j) Copeoglossum nigropunctatum; k) Uracentron azureum guentheri; 1) Uranoscodon superciliosus; m) Lepidoblepharis heyerorum; n) Plica umbra; o) Chironius scurrulus; p) Micrurus langsdorffii; q) Tantilla melanocephala; r) Dipsas catesbyi; s) Oxyrhopus occipitalis; t) Bothriopsis taeniata; u) Liophis typhlus; v) Dendrophidion dendrophis; w) Dipsas indica; x) Taeniophallus aff. occipitalis; y) Bothrops atrox; z) Imantodes cenchoa (Photo i - B. Marioni, too photos - F. Waldez).

grandes áreas de florestas de terras baixas na Amazônia, são requeridos estudos de longo prazo com grande esforço de amostragem (ver Rodríguez \& Duellman 1994, Duellman 1999, 2005, Donnelly et al. 2005, Lima et al. 2008, Vitt et al. 2008). Nestes ambientes florestais, as serpentes normalmente correspondem ao grupo da herpetofauna menos conhecido por dificuldades associadas à detecção das espécies (Martins \& Oliveira 1998, Bernarde \& Abe 2006).

De fato, a amostragem da diversidade da herpetofauna no baixo Purus se mostrou satisfatória para sapos e lagartos nas florestas de terra firme e de várzea, com eficiência menor na amostragem de serpentes nos dois tipos de florestas (ver Figura 5). No entanto, alguns taxa foram notadamente ausentes na composição das espécies observada. No geral, foi baixo o número de registros de espécies com hábitos aquático e fossorial. Não foram observados répteis Squamata fossoriais das famílias Amphisbaenidae (Amphisbaena spp.) e Gymnophthalmidae (Bachia spp.); também ausentes foram lagartos dos gêneros Neusticurus semi-aquático (Gymnophthalmidae), Stenocercus terrícola (Tropiduridae) e Polychrus arborícola 
Tabela 2. Espécies de répteis Squamata encontradas na região do baixo rio Purus, Amazônia Central, Brasil. O número de espécimes foi indicado por: tipo de ambiente (floresta de terra firme e floresta de várzea), tipo de unidade amostral (parcela e transecto) e métodos de coleta (armadilha de interceptação com funis duplos - AIF, armadilha de interceptação e queda - AIQ, procura limitada por tempo - PLT e registro ocasional).

Table 2. Species of Squamata reptilians found in the lower Purus River Basin, Central Amazonia, Brazil. The individual numbers are indicated by: type of forests habitat (terra firmenonflooded forests and várzea flooded forests), sampling unit (plot and transect) and sampling method (time constrained audio-visual search - PLT, pitfall traps with drift fence - AIQ, double-ended funnel traps with drift fence - AIF and occasional records).

\begin{tabular}{|c|c|c|c|c|c|c|c|c|c|}
\hline \multirow[t]{2}{*}{ REPTILIA : SQUAMATA (85 spp.) } & \multirow[t]{2}{*}{ Total. } & \multicolumn{2}{|c|}{$\begin{array}{c}\text { Parcelas várzea } \\
(\mathbf{n}=\mathbf{2 8})\end{array}$} & \multicolumn{2}{|c|}{$\begin{array}{l}\text { Parcelas terra } \\
\text { firme }(\mathrm{n}=32)\end{array}$} & \multicolumn{3}{|c|}{$\begin{array}{l}\text { Transectos terra firme } \\
\qquad(\mathrm{n}=5)\end{array}$} & \multirow{2}{*}{$\begin{array}{l}\text { Registros } \\
\text { ocasionais }\end{array}$} \\
\hline & & AIF & PLT & AIF & PLT & AIQ & AIF & PLT & \\
\hline Subordem SAURIA (34 spp.) & 1996 & 3 & 447 & 58 & 792 & 187 & 37 & 462 & 34 \\
\hline \multicolumn{10}{|l|}{ Iguanidae (1 sp.) } \\
\hline Iguana iguana (Linnaeus, 1758) & 7 & 0 & 0 & 0 & 1 & 2 & 0 & 3 & 1 \\
\hline \multicolumn{10}{|l|}{ Polychrotidae (5 spp.) } \\
\hline Anolis fuscoauratus D’Orbigny, 1837 & 219 & 0 & 65 & 0 & 65 & 19 & 0 & 70 & 0 \\
\hline Anolis tandai (Avila-Pires, 1995) & 45 & 0 & 1 & 2 & 16 & 4 & 2 & 20 & 0 \\
\hline Anolis ortonii Cope, 1868 & 4 & 0 & 2 & 0 & 0 & 2 & 0 & 0 & 0 \\
\hline Anolis punctatus Daudin, 1802 & 4 & 0 & 1 & 0 & 1 & 1 & 0 & 1 & 0 \\
\hline Anolis transversalis Duméril, 1851 & 5 & 0 & 0 & 0 & 2 & 2 & 0 & 1 & 0 \\
\hline \multicolumn{10}{|l|}{ Tropiduridae (4 spp.) } \\
\hline Plica plica (Linnaeus, 1758) & 12 & 0 & 0 & 0 & 4 & 3 & 0 & 5 & 0 \\
\hline Plica umbra (Linnaeus, 1758) & 24 & 0 & 11 & 0 & 10 & 1 & 0 & 2 & 0 \\
\hline Uracentron azureum (Linnaeus, 1758) & 2 & 0 & 0 & 0 & 0 & 0 & 0 & 1 & 1 \\
\hline Uranoscodon superciliosus (Linnaeus, 1758) & 125 & 0 & 72 & 0 & 32 & 1 & 0 & 16 & 4 \\
\hline \multicolumn{10}{|l|}{ Gekkonidae (1 sp.) } \\
\hline Hemidactylus mabouia (Moreau de Jonnès, 1818) & 2 & 0 & 0 & 0 & 0 & 0 & 0 & 0 & 2 \\
\hline \multicolumn{10}{|l|}{ Phyllodactylidae (1 sp.) } \\
\hline Thecadactylus solimoensis Bergmann \& Russell, 2007 & 7 & 0 & 0 & 0 & 1 & 0 & 0 & 5 & 1 \\
\hline \multicolumn{10}{|l|}{ Sphaerodactylidae (4 spp.) } \\
\hline Chatogekko amazonicus (Andersson, 1918) & 303 & 0 & 2 & 22 & 205 & 25 & 3 & 46 & 0 \\
\hline Gonatodes hasemani Griffin, 1917 & 4 & 0 & 0 & 0 & 3 & 0 & 1 & 0 & 0 \\
\hline Gonatodes humeralis (Guichenot, 1855) & 348 & 0 & 76 & 0 & 151 & 0 & 0 & 121 & 0 \\
\hline Lepidoblepharis heyerorum Vanzolini, 1978 & 1 & 0 & 0 & 1 & 0 & 0 & 0 & 0 & 0 \\
\hline \multicolumn{10}{|l|}{ Teiidae (6 spp.) } \\
\hline Ameiva ameiva (Linnaeus, 1758) & 250 & 0 & 30 & 17 & 145 & 20 & 9 & 29 & 0 \\
\hline Crocodilurus amazonicus Spix, 1825 & 34 & 0 & 1 & 0 & 1 & 0 & 0 & 32 & 0 \\
\hline Dracaena guianensis Daudin, 1802 & 1 & 0 & 0 & 0 & 0 & 0 & 0 & 0 & 1 \\
\hline Kentropyx altamazonica (Cope, 1876) & 131 & 0 & 99 & 0 & 0 & 0 & 0 & 32 & 0 \\
\hline Kentropyx pelviceps Cope, 1868 & 99 & 0 & 14 & 2 & 40 & 11 & 7 & 25 & 0 \\
\hline Tupinambis teguixin (Linnaeus, 1758) & 62 & 0 & 28 & 0 & 28 & 0 & 0 & 6 & 0 \\
\hline \multicolumn{10}{|l|}{ Gymnophthalmidae (10 spp.) } \\
\hline Alopoglossus angulatus (Linnaeus, 1758) & 21 & 0 & 0 & 0 & 0 & 12 & 4 & 5 & 0 \\
\hline Alopoglossus aff. atriventris Duellman, 1973 & 88 & 0 & 0 & 8 & 43 & 22 & 5 & 10 & 0 \\
\hline Arthrosaura reticulata (O’Shaughnessy, 1881) & 50 & 2 & 0 & 3 & 14 & 20 & 3 & 8 & 0 \\
\hline Cercosaura argulus Peters, 1863 & 3 & 0 & 0 & 0 & 0 & 3 & 0 & 0 & 0 \\
\hline Cercosaura ocellata Wagler, 1830 & 1 & 0 & 0 & 1 & 0 & 0 & 0 & 0 & 0 \\
\hline Iphisa elegans Gray, 1851 & 11 & 0 & 0 & 0 & 1 & 9 & 0 & 1 & 0 \\
\hline Leposoma osvaldoi Ávila-Pires, 1995 & 3 & 0 & 0 & 0 & 0 & 3 & 0 & 0 & 0 \\
\hline Leposoma percarinatum (Müller, 1923) & 23 & 0 & 1 & 1 & 8 & 13 & 0 & 0 & 0 \\
\hline Leposoma snethlageae Ávila-Pires, 1995 & 1 & 0 & 0 & 0 & 0 & 1 & 0 & 0 & 0 \\
\hline Ptychoglossus brevifrontalis Boulenger, 1912 & 5 & 0 & 0 & 0 & 0 & 5 & 0 & 0 & 0 \\
\hline \multicolumn{10}{|l|}{ Scincidae (2 spp.) } \\
\hline Varzea bistriata (Spix, 1825) & 2 & 0 & 0 & 0 & 0 & 2 & 0 & 0 & 0 \\
\hline Copeoglossum nigropunctatum (Spix, 1825) & 99 & 1 & 44 & 1 & 21 & 6 & 3 & 23 & 0 \\
\hline Subordem OPHIDIA (51 spp.) & 177 & 5 & 31 & 4 & 42 & 32 & 25 & 13 & 25 \\
\hline Leptotyphlopidae (1 sp.) & . & & & & & & & & \\
\hline Epictia sp. & 1 & 0 & 0 & 0 & 0 & 1 & 0 & 0 & 0 \\
\hline
\end{tabular}


Waldez, F. et al.

Tabela 2. Continuação...

\begin{tabular}{|c|c|c|c|c|c|c|c|c|c|}
\hline \multirow[t]{2}{*}{ REPTILIA : SQUAMATA (85 spp.) } & \multirow[t]{2}{*}{ Total. } & \multicolumn{2}{|c|}{$\begin{array}{c}\text { Parcelas várzea } \\
(\mathbf{n}=\mathbf{2 8}) \\
\end{array}$} & \multicolumn{2}{|c|}{$\begin{array}{l}\text { Parcelas terra } \\
\text { firme }(\mathrm{n}=\mathbf{3 2})\end{array}$} & \multicolumn{3}{|c|}{$\begin{array}{l}\text { Transectos terra firme } \\
\qquad(\mathrm{n}=5)\end{array}$} & \multirow{2}{*}{$\begin{array}{l}\text { Registros } \\
\text { ocasionais }\end{array}$} \\
\hline & & AIF & PLT & AIF & PLT & AIQ & AIF & $\begin{array}{l}\text { PLT } \\
\end{array}$ & \\
\hline \multicolumn{10}{|l|}{ Typhlopidae (2 spp.) } \\
\hline Typhlops minuisquamus Dixon \& Hendricks, 1979 & 1 & 0 & 0 & 0 & 0 & 1 & 0 & 0 & 0 \\
\hline Typhlops reticulatus (Linnaeus, 1758) & 1 & 0 & 0 & 0 & 0 & 1 & 0 & 0 & 0 \\
\hline \multicolumn{10}{|l|}{ Aniliidae (1 sp.) } \\
\hline Anilius scytale (Linnaeus, 1758) & 3 & 1 & 0 & 0 & 0 & 0 & 2 & 0 & 0 \\
\hline \multicolumn{10}{|l|}{ Boidae (5 spp.) } \\
\hline Boa constrictor Linnaeus, 1758 & 2 & 0 & 0 & 0 & 0 & 0 & 0 & 0 & 2 \\
\hline Corallus caninus (Linnaeus, 1758) & 1 & 0 & 0 & 0 & 0 & 0 & 0 & 0 & 1 \\
\hline Corallus hortulanus (Linnaeus, 1758) & 8 & 0 & 3 & 0 & 2 & 0 & 0 & 2 & 1 \\
\hline Epicrates cenchria (Linnaeus, 1758) & 2 & 0 & 0 & 0 & 1 & 1 & 0 & 0 & 0 \\
\hline Eunectes murinus (Linnaeus, 1758) & 3 & 0 & 1 & 0 & 0 & 0 & 0 & 0 & 2 \\
\hline \multicolumn{10}{|l|}{ Colubridae (12 spp.) } \\
\hline Chironius carinatus (Linnaeus, 1758) & 1 & 0 & 0 & 0 & 1 & 0 & 0 & 0 & 0 \\
\hline Chironius exoletus (Linnaeus, 1758) & 1 & 0 & 0 & 0 & 1 & 0 & 0 & 0 & 0 \\
\hline Chironius fuscus (Linnaeus, 1758) & 4 & 1 & 1 & 0 & 0 & 1 & 1 & 0 & 0 \\
\hline Chironius scurrulus (Wagler, 1824) & 6 & 0 & 3 & 0 & 3 & 0 & 0 & 0 & 0 \\
\hline Dendrophidion dendrophis (Schlegel, 1837) & 2 & 1 & 0 & 0 & 1 & 0 & 0 & 0 & 0 \\
\hline Drymoluber dichrous (Peters, 1863) & 4 & 0 & 0 & 0 & 3 & 0 & 1 & 0 & 0 \\
\hline Leptophis ahaetulla (Linnaeus, 1758) & 1 & 0 & 0 & 0 & 0 & 0 & 0 & 0 & 1 \\
\hline Oxybelis aeneus (Wagler, 1824) & 1 & 0 & 0 & 0 & 0 & 0 & 0 & 0 & 1 \\
\hline Oxybelis fulgidus (Daudin, 1803) & 2 & 0 & 0 & 0 & 1 & 0 & 0 & 0 & 1 \\
\hline Pseustes poecilonotus (Günther, 1858) & 2 & 0 & 0 & 0 & 1 & 0 & 0 & 1 & 0 \\
\hline Spilotes pullatus (Linnaeus, 1758) & 2 & 0 & 1 & 0 & 0 & 0 & 0 & 1 & 0 \\
\hline Tantilla melanocephala (Linnaeus, 1758) & 2 & 0 & 0 & 0 & 2 & 0 & 0 & 0 & 0 \\
\hline \multicolumn{10}{|l|}{ Dipsadidae (20 spp.) } \\
\hline Atractus poeppigi (Jan, 1862) & 4 & 0 & 0 & 0 & 0 & 2 & 2 & 0 & 0 \\
\hline Atractus schach (Boie, 1827) & 2 & 0 & 0 & 0 & 0 & 2 & 0 & 0 & 0 \\
\hline $\begin{array}{l}\text { Atractus torquatus (Duméril, Bibron \& Duméril, } \\
\text { 1854) }\end{array}$ & 24 & 0 & 0 & 0 & 0 & 16 & 6 & 0 & 2 \\
\hline Dipsas catesbyi (Sentzen, 1796) & 7 & 0 & 6 & 0 & 1 & 0 & 0 & 0 & 0 \\
\hline Dipsas indica Laurenti, 1768 & 3 & 0 & 3 & 0 & 0 & 0 & 0 & 0 & 0 \\
\hline Drepanoides anomalus (Jan, 1863) & 4 & 1 & 0 & 1 & 0 & 0 & 2 & 0 & 0 \\
\hline Helicops angulatus (Linnaeus, 1758) & 4 & 0 & 0 & 0 & 2 & 2 & 0 & 0 & 0 \\
\hline $\begin{array}{l}\text { Hydrodynastes gigas (Duméril, Bibron \& Duméril, } \\
\text { 1854) }\end{array}$ & 2 & 0 & 0 & 0 & 0 & 0 & 0 & 0 & 2 \\
\hline Hydrops martii (Wagler, 1824) & 1 & 0 & 0 & 0 & 0 & 0 & 0 & 0 & 1 \\
\hline Imantodes cenchoa (Linnaeus, 1758) & 17 & 0 & 6 & 0 & 6 & 0 & 0 & 4 & 1 \\
\hline Leptodeira annulata (Linnaeus, 1758) & 4 & 0 & 0 & 0 & 1 & 0 & 0 & 3 & 0 \\
\hline Liophis typhlus (Linnaeus, 1758) & 4 & 0 & 1 & 0 & 1 & 0 & 0 & 1 & 1 \\
\hline Oxyrhopus occipitalis Wagler in Spix, 1824 & 2 & 0 & 0 & 0 & 1 & 0 & 1 & 0 & 0 \\
\hline Oxyrhopus vanidicus Lynch, 2009 & 3 & 0 & 0 & 0 & 1 & 0 & 2 & 0 & 0 \\
\hline Philodryas argentea (Daudin, 1803) & 1 & 0 & 1 & 0 & 0 & 0 & 0 & 0 & 0 \\
\hline Pseudoboa coronata Schneider, 1801 & 2 & 0 & 0 & 0 & 0 & 0 & 2 & 0 & 0 \\
\hline Siphlophis cervinus (Laurenti, 1768) & 1 & 0 & 0 & 0 & 0 & 0 & 0 & 0 & 1 \\
\hline Taeniophallus aff. occipitalis (Jan, 1863) & 2 & 0 & 0 & 1 & 0 & 0 & 1 & 0 & 0 \\
\hline Thamnodynastes pallidus (Linnaeus, 1758) & 2 & 1 & 1 & 0 & 0 & 0 & 0 & 0 & 0 \\
\hline Xenopholis scalaris (Wucherer, 1861) & 5 & 0 & 1 & 1 & 1 & 0 & 1 & 1 & 0 \\
\hline \multicolumn{10}{|l|}{ Elapidae (6 spp.) } \\
\hline Micrurus filiformis (Günther, 1859) & 1 & 0 & 1 & 0 & 0 & 0 & 0 & 0 & 0 \\
\hline Micrurus hemprichii (Jan, 1858) & 7 & 0 & 0 & 0 & 0 & 5 & 2 & 0 & 0 \\
\hline Micrurus langsdorffii Wagler, 1824 & 1 & 0 & 0 & 1 & 0 & 0 & 0 & 0 & 0 \\
\hline Micrurus lemniscatus (Linnaeus, 1758) & 1 & 0 & 0 & 0 & 1 & 0 & 0 & 0 & 0 \\
\hline
\end{tabular}


Tabela 2. Continuação...

\begin{tabular}{|c|c|c|c|c|c|c|c|c|c|}
\hline \multirow[t]{2}{*}{ REPTILIA : SQUAMATA (85 spp.) } & \multirow[t]{2}{*}{ Total. } & \multicolumn{2}{|c|}{$\begin{array}{c}\text { Parcelas várzea } \\
(\mathbf{n}=\mathbf{2 8})\end{array}$} & \multicolumn{2}{|c|}{$\begin{array}{l}\text { Parcelas terra } \\
\text { firme }(\mathrm{n}=\mathbf{3 2})\end{array}$} & \multicolumn{3}{|c|}{$\begin{array}{l}\text { Transectos terra firme } \\
(n=5)\end{array}$} & \multirow{2}{*}{$\begin{array}{l}\text { Registros } \\
\text { ocasionais }\end{array}$} \\
\hline & & AIF & PLT & AIF & PLT & AIQ & AIF & PLT & \\
\hline Micrurus spixii Wagler, 1824 & 1 & 0 & 0 & 0 & 0 & 0 & 0 & 0 & 1 \\
\hline Micrurus surinamensis (Cuvier, 1817) & 1 & 0 & 0 & 0 & 0 & 0 & 0 & 0 & 1 \\
\hline \multicolumn{10}{|l|}{ Viperidae (4 spp.) } \\
\hline Bothriopsis bilineata (Wied, 1825) & 1 & 0 & 0 & 0 & 0 & 0 & 0 & 0 & 1 \\
\hline Bothriopsis taeniata (Wagler, 1824) & 2 & 0 & 0 & 0 & 2 & 0 & 0 & 0 & 0 \\
\hline Bothrops atrox (Linnaeus, 1758) & 17 & 0 & 2 & 0 & 9 & 0 & 2 & 0 & 4 \\
\hline Lachesis muta (Linnaeus, 1766) & 1 & 0 & 0 & 0 & 0 & 0 & 0 & 0 & 1 \\
\hline
\end{tabular}

Tabela 3. Riqueza de espécies da herpetofauna na região do baixo rio Purus, Amazonas, Brasil. O número de espécies registradas encontra-se apresentado por método de coleta, tipo de floresta e estratégia de amostragem. Entre parênteses o número de espécies registradas exclusivamente por cada tipo de método de coleta.

Table 3. Richness of herpetofauna species from the lower Purus River Basin, Central Amazonia, Brazil. The number of species is indicated by type of forest habitat, sampling unit and sampling method. The number of species recorded exclusively by each sampling method is noted in brackets.

\begin{tabular}{|c|c|c|c|c|}
\hline \multirow[b]{2}{*}{ Grupo } & \multirow[b]{2}{*}{ método de coleta } & \multicolumn{3}{|c|}{ Número de espécie registradas (registradas apenas pelo método) } \\
\hline & & $\begin{array}{c}\text { Parcelas de } \\
\text { várzea }\end{array}$ & $\begin{array}{l}\text { Parcelas de } \\
\text { terra firme }\end{array}$ & $\begin{array}{l}\text { Transectos de } \\
\text { terra firme }\end{array}$ \\
\hline \multirow[t]{4}{*}{ ANFÍBIOS } & Armadilha de interceptação com funis - AIF & $4(0)$ & $6(0)$ & $15(0)$ \\
\hline & Procura limitada por tempo - PLT & $36(32)$ & $48(42)$ & $31(14)$ \\
\hline & Armadilhas de interceptação e queda - AIQ & - & - & $27(10)$ \\
\hline & Total de espécies registradas & 36 & 48 & 45 \\
\hline \multirow[t]{4}{*}{ LAGARTOS } & Armadilha de interceptação com funis - AIF & $2(1)$ & $10(2)$ & $9(1)$ \\
\hline & Procura limitada por tempo - PLT & $16(15)$ & $21(14)$ & $22(6)$ \\
\hline & Armadilhas de interceptação e queda - AIQ & - & - & $23(7)$ \\
\hline & Total de espécies registradas & 17 & 23 & 30 \\
\hline \multirow[t]{4}{*}{ SERPENTES } & Armadilha de interceptação com funis - AIF & $5(3)$ & $4(3)$ & $13(7)$ \\
\hline & Procura limitada por tempo - PLT & $14(12)$ & $21(20)$ & $7(6)$ \\
\hline & Armadilhas de interceptação e queda - AIQ & - & - & $10(6)$ \\
\hline & Total de espécies registradas & 17 & 24 & 24 \\
\hline
\end{tabular}

(Polychrotidae). Todos estes gêneros apresentam espécies ocorrendo na região da bacia do rio Purus (Rodríguez 2003, Avila-Pires et al. 2009). Também, salamandras da família Plethodontidae (Bolitoglossa spp.) não foram encontradas apesar de ocorrerem em ambos os interflúvios do rio Purus, como indicam espécimes depositados na Coleção de Anfíbios e Répteis do INPA. De acordo com esses resultados, acreditamos que a estratégia de amostragem empregada foi eficaz em representar a maior parte da diversidade da herpetofauna terrestre de hábitos arborícola e terrícola.

Novos registros de espécies foram feitos para os sapos Dendropsophus allenorum, primeiro registro para o Brasil (ver Frost 2011), e Scinax pedromedinae, segundo registro no país (ver Hoogmoed \& Avila-Pires 2011). Estas duas espécies arborícolas são conhecidas da Amazônia Peruana, Boliviana e recentemente Brasileira, para o alto rio Amazonas/Solimões e nas bacias dos rios Madre de Dios e Purus (Rodríguez \& Duellman 1994, Duellman 1999, Ron 2000, Hoogmoed \& Avila-Pires 2011, Frost 2011). Na região do baixo rio Purus D. allenorum (Figura 4c) e S. pedromedinae (Figura 41) ocorreram associadas às florestas de várzeas.

Comparando a eficiência dos métodos de coleta utilizados (Tabela 3), a busca ativa realizada por coletores capacitados (procura limitada por tempo - PLT) foi o método mais eficaz na amostragem de um maior número de espécies da herpetofauna, como já descrito em inventários em regiões florestais na Amazônia (Neckel-Oliveira \& Gordo 2004, Donnelly et al. 2005, Vogt et al. 2007, Turci \& Bernarde 2008). O uso de armadilhas de interceptação e queda - AIQ (pitfall traps) apesar de bastante indicado para amostragem da herpetofauna em ambientes de florestas tropicais (Cechin \& Martins 2000, Ribeiro-Júnior et al. 2008) é de difícil utilização em áreas com solos hidricamente saturados, como nas florestas sazonalmente alagáveis (Neckel-Oliveira \& Gordo 2004). Dentro de uma estratégia que privilegia a amostragem de um grande número de localidades, o uso de pitfall traps onera as relações de custo-benefício. Neste estudo, as amostragens com AIQ foram restritas a um menor número de localidades de floresta de terra firme e utilizadas por um período maior de tempo de abertura (ver Estratégias de amostragem). As armadilhas de interceptação com funis duplos - AIF (double-ended funnel traps), por serem de rápida instalação, foram intermediárias nas relações de custos-benefício para espacializar muitas unidades amostrais em uma grande área. A instalação de funnel traps é facilmente adaptável aos solos hidromórficos das florestas de várzea. Neste e em outros estudos realizados em ambientes florestais as AIF foram eficientes na captura serpentes (Vogt et al. 2007, Bateman et al. 2009), um dos grupos menos representados em inventários rápidos (Martins \& Oliveira 1998, 
Waldez, F. et al.
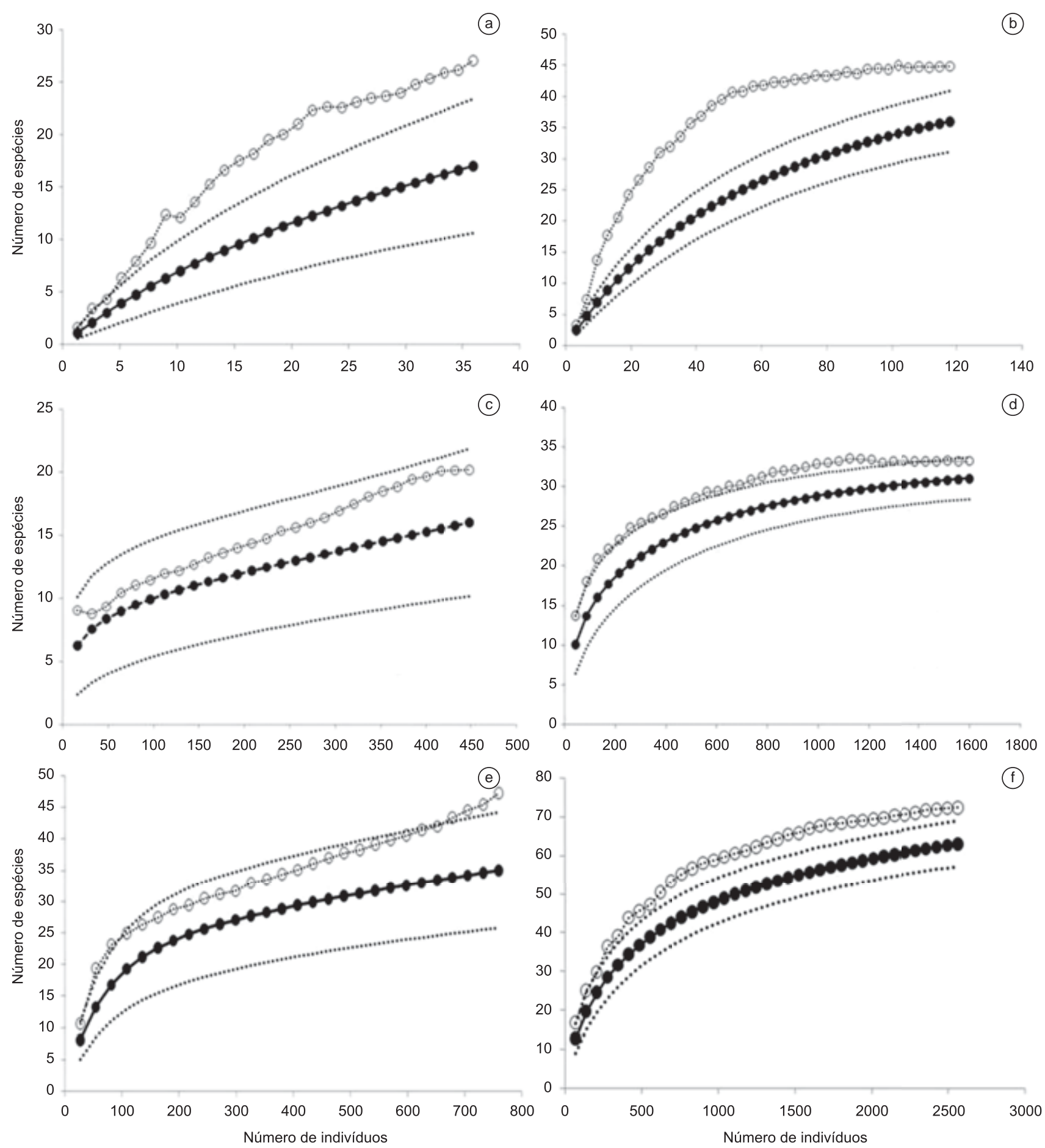

Figura 5. Curvas de rarefação baseadas no número de indivíduos (círculos cheios) para as comunidades de serpentes (a, b), lagartos (c, d) e anfíbios (e, f) da região do baixo rio Purus, Amazônia Central, Brasil. As linhas tracejadas representam intervalos de confiança de $95 \%$. Os círculos abertos demonstram a média das estimativas de riqueza de quatro diferentes estimadores (ACE, Chao 1, Jacknife \& Bootstrap). As comunidades de espécies das florestas de várzea encontram-se na coluna esquerda e das espécies de florestas de terra firme na coluna direita.

Figure 5. Abundance-based rarefaction curves (Black symbols) for the assemblages of snakes (a, b), lizards (c, d) and amphibians (e, f) from the lower Purus River Basin in Central Amazonia, Brazil. Dotted lines denote $95 \%$ confidence intervals. Clear symbol lines show the mean estimated richness from four different richness estimators (ACE, Chao 1, Jacknife \& Bootstrap). The species assemblages from the varzea flooded forests are in the left column and species from the terra firme nonflooded forests in the right column. 


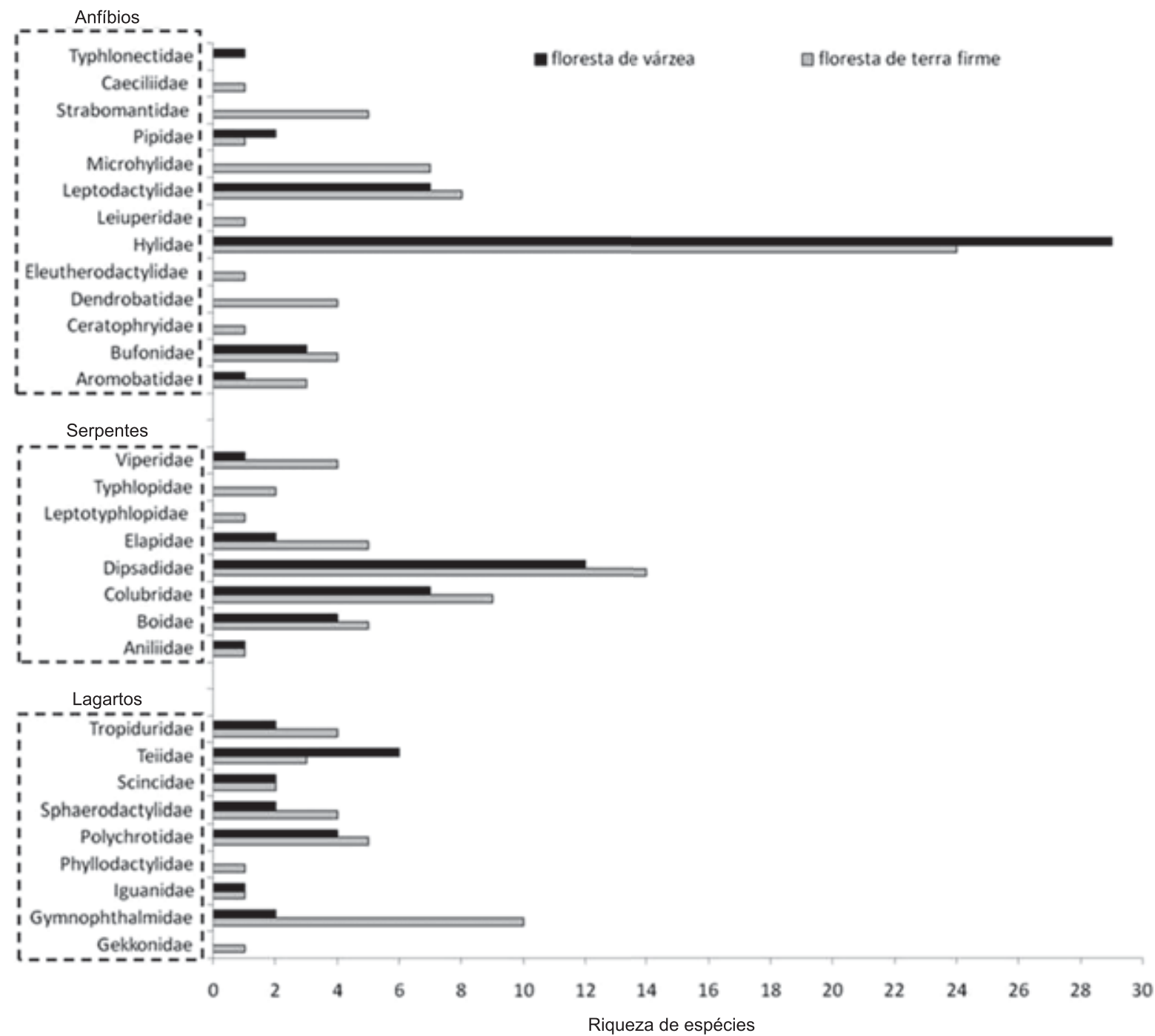

Figura 6. Número de espécies por famílias de anfíbios e répteis Squamata (lagartos e serpentes) encontradas nas florestas de terra firme e florestas de várzea da região do baixo rio Purus, Amazônia Central, Brasil.

Figure 6. Number of species by families of amphibians and Squamata reptilians (lizards and snakes) found in the terra firme nonflooded forests and the varzea flooded forests from the lower Purus River Basin, Central Amazonia, Brazil.

Bernarde \& Abe 2006). Considerando nossos resultados (Tabela 3), julgamos adequado utilizar AIF como método complementar a busca ativa por coletores capacitados, para amostragem da herpetofauna em florestas tropicais de planícies de terra firme e sazonalmente alagáveis. No entanto, resultados conflitantes quanto à eficiência das funnel traps na amostragem da herpetofauna são descritos na literatura (ver Ribeiro-Júnior et al. 2008). Estas diferenças podem estar relacionadas com características dos habitas. Nas florestas de várzea, certamente a menor eficácia relativa dos funnel traps foi influenciada pela menor representatividade de grupos fossoriais e de serrapilheira presentes na terra firme (ver Figura 6). Também, podem decorrer de variações na metodologia de uso dos funnel traps. Para ambientes florestais, independente da disposição dos conjuntos de AIF (linhas retas, linhas em Y, etc), recomendamos o uso de funis com dupla abertura, fixados à cerca guia, parcialmente enterrados e com as entradas preenchidas com o substrato do solo (ver Figura 2e, f).

Além deste estudo, poucas pesquisas realizaram análises amplas sobre a diversidade da herpetofauna entre florestas sazonalmente alagáveis e de florestas de terra firme na Amazônia (ver Crump 1971, Doan \& Arizábal 2002, Neckel-Oliveira \& Gordo 2004, Duellman 2005). Alguns estudos realizaram uma análise restrita para sapos (Gascon 1996, Gascon et al. 2000, Gordo 2003). Em todos, foi comum uma distribuição desigual do esforço de amostragem devido a diferente a disponibilidade em área de cada tipo de ambiente. Os habitats de florestas de terra firme tiveram amostragem priorizada pela maior complexidade estrutural e pelo uso de armadilhas de interceptação e queda (AIQ) que são de difícil instalação em solos úmidos como das florestas alagáveis (Neckel-Oliveira \& Gordo 2004).

De maneira geral, uma maior diversidade de espécies da herpetofauna tem sido associada às florestas de terra firme (Gascon 1996, Gascon et al. 2000, Doan \& Arizábal 2002, Neckel-Oliveira \& Gordo 2004, Duellman 2005). Em alguns estudos têm-se encontrado nas florestas de várzea uma diversidade maior ou similar a das florestas de terra firme (Crump 1971, Gordo 2003).

Na região do baixo Purus, foi encontrada uma maior riqueza de espécies e de famílias nas florestas de terra firme que nas florestas de 
várzea com marcante ausência de espécies fossoriais e de serrapilheira (Figura 6). No entanto, nas florestas de várzea encontramos um maior número de espécies de sapos arborícolas (Hylidae) e de lagartos heliotérmicos de maior porte (Teiidae) (Figura 6), com ocorrência de espécies semi-aquáticas pouco registradas (e.g. Dracaena guianensis) (Tabela 2, Figura 4i).

Uma riqueza de espécies da herpetofauna mais parecida em números absolutos entre os diferentes tipos de paisagens florestais pode ser observada considerando o número de espécies entre períodos de amostragem que empregaram esforço de coleta e estratégias de amostragem mais similares (ver Tabela 3). No entanto, apesar poderem ser similares em número espécies de anfíbios e répteis Squamata, as florestas de terra firme e de várzea na região estudada foram diferentes quanto ao conjunto de espécies. As várzeas foram amostradas durante a fase terrestre e a ausência de várias famílias de espécies da herpetofauna encontradas nos solos das florestas de terra firme adjacentes, indicou forte efeito do pulso de inundação (Junk et al. 1989) na estruturação das comunidades de anfíbios e répteis nas florestas de várzea. De fato, para florestas sazonalmente inundáveis na Amazônia Central o pulso de inundação tem sido reportado como o principal evento ecológico e histórico estruturador das comunidades animal e vegetal (ver Junk 1997, Haugaasen \& Peres 2005).

A diversidade complementar de espécies de anfíbios e répteis Squamata entre as florestas de terra firme e as florestas de várzea (diversidade beta- $\beta$ ), relacionada com a maior abrangência local destas paisagens florestais, possivelmente representa a maior contribuição para a diversidade regional da herpetofauna no baixo rio Purus (diversidade Gama- $\gamma$ ).

\section{Agradecimentos}

A Fundação de Amparo à Pesquisa do Estado do Amazonas (FAPEAM) concedeu bolsa de doutorado para F. Waldez (POSGRAD 001/2008). Ao CNPq pela Bolsa de Produtividade em Pesquisa concedida para M. Menin e R. C. Vogt. O Centro Estadual de Unidades de Conservação (CEUC) da Secretaria de Estado de Meio Ambiente e Desenvolvimento Sustentável do Amazonas (SDS) autorizou a pesquisa na Reserva de Desenvolvimento Sustentável PiagaçuPurus (nº proc.: 39/2010 SDS/CEUC). Os resultados apresentados neste estudo foram financiados pela FAPEAM através do Programa Integrado de Pesquisa e Inovação Tecnológica (PIPT) concedido para R. C. Vogt (nº proc.: 877/2003). Também pelo Instituto Piagaçu (IPI) em parceria com a Wildlife Conservation Society (WCS). Hermogenes Costa Neto construiu o mapa da Figura 1. Dois revisores anônimos fizeram relevantes contribuições à primeira versão do manuscrito.

\section{Referências Bibliográficas}

ALBERNAZ, A.L.K.M. \& VENTICINQUE, E.M. 2003. Reserva de Desenvolvimento Sustentável Piagaçu-Purus: Características e Limites Geográficos In Piagaçu-Purus: Bases Científicas para Criação de uma Reserva de Desenvolvimento Sustentável (C.P. de Deus, R. Da Silveira \& L.H.R. Py-Daniel, eds). IDSM, Manaus, p.3-12

ALLMON, W.D. 1991. A plot study of forest floor litter frogs, Central Amazon, Brazil. J. Trop. Ecol. 7:503-22. http://dx.doi.org/10.1017/ S0266467400005885

AVILA-PIRES, T.C.S. 1995. Lizards of Brazilian Amazonia (Reptilia: Squamata). Zool. Verh. 299:1-706.

AVILA-PIRES, T.C.S., VITT, L.J., SARTORIUS, S.S. \& ZANI, P.A. 2009. Squamata (Reptilia) from four sites in southern Amazonia, with a biogeographic analysis of Amazonian lizards. Bol. Mus. Para. Emílio Goeldi. Cienc. Nat. 4(2):99-118.
AVILA-PIRES, T.C.S., HOOGMOED, M.S. \& ROCHA, W.A. 2010. Notes on the Vertebrates of northern Pará, Brazil: a forgotten part of the Guianan Region, I. Herpetofauna. Bol. Mus. Para. Emilio Goeldi Cienc. Nat. 5(1):13-112.

AZEVEDO-RAMOS, C. \& GALATTI, U. 2001. Patterns of amphibian diversity in Brazilian Amazonia: conservation implications. Biol. Conserv. 103:103-111. http://dx.doi.org/10.1016/S00063207(01)00129-X

BATEMAN, L.H., CHUNG-MacCOUBREY, A., SNELL, H.L. \& FINCH, D.M. 2009. Abundance and species richness of snakes along the Middle Rio Grande riparian forest in New Mexico. Herpetol. Conserv. Biol. 4(1):1-8.

BERNARDE, P.S. \& ABE, A.S. 2006. A snake community at Espigão do Oeste, Rondônia, Southwestern Amazon, Brazil. South Am. J. Herpetol. 1(2):102-113. http://dx.doi.org/10.2994/18089798(2006)1[102:ASCAED]2.0.CO;2

BERNARDE, P.S. \& MACEDO, L.C. 2008. Impacto do desmatamento e formação de pastagens sobre a anurofauna de serapilheira em Rondônia. Iheringia, Sér. Zool. 98(4):454-459.

BERNARDE, P. S., MACHADO, R.A. \& TURCI, L.C.B. 2011. Herpetofauna da área do Igarapé Esperança na Reserva Extrativista Riozinho da Liberdade, Acre - Brasil. Biota Neotrop. 11(3): http://www. biotaneotropica.org.br/v11n3/en/abstract?inventory+bn02111032011 (último acesso em 14/02/2012).

BRASIL. Ministério do Meio Ambiente. 2001. Avaliação e identificação de ações prioritárias para a conservação, utilização sustentável e repartição dos benefícios da biodiversidade da Amazônia brasileira. Ministério do Meio Ambiente, Brasília.

CECHIN, S.Z. \& MARTINS, M. 2000. Eficiência de armadilhas de queda (pitfall traps) em amostragens de anfíbios e répteis no Brasil. Rev. Bras. Zool. 17:729-740. http://dx.doi.org/10.1590/S0101-81752000000300017

COLWELL, R.K. 2004. EstimateS: statistical estimation of species richness and shared species from samples. version 7. User's guide and application. University of Connecticut, Storrs. http://viceroy.eeb.uconn.edu/estimates (último acesso em 14/12/2011).

CRACRAFT, J. 1985. Historical biogeography and patterns of differentiation within the South American avifauna: areas of endemism. Ornith. Mono. 36:49-84. http://dx.doi.org/10.2307/40168278

CRUMP, M.L. \& SCOTT, N.J. 1994. Visual encounter surveys. In Measuring and monitoring biological diversity: standard methods for amphibians (W.R Heyer, M.A. Donnelly, R.W. McDiarmid, L.A.C. Hayek \& M.S. Foster, eds). Smithsonian Institution Press, Washington, p.84-92.

CRUMP, M.L. 1971. Quantitative analysis of the ecological distribution of a tropical herpetofauna. Occas. Pap. Univ. Kansas Mus. Nat. Hist. 3:1-62.

DOAN, T.M. 2003. Which methods are most effective for surveying rain forest herpetofauna? J. Herpet. 37:72-81. http://dx.doi.org/10.1670/00221511(2003)037[0072:WMAMEF]2.0.CO;2

DOAN, T.M. \& ARIZÁBALA.W. 2002. Microgeographic variation in species composition of the herpetofaunal communities of Tambopata Region, Peru. Biotropica. 34(1):101-117.

DONNELLY, M.A., CHEN, M.H., \& WATKINS, G.G. 2005. Sampling amphibians and reptiles in the Iwokrama Forest ecosystem. Proc. Acad. Nat. Sci. Philadelphia. 154(1):55-69. http://dx.doi.org/10.1635/00973157(2004)154[0055:SAARIT]2.0.CO;2

DUELLMAN, W.E. 1990. Herpetofaunas in Neotropical rainforests: comparative composition, history, and resource use. In Four Neotropical Rainforests (A.H. Gentry, ed.). Yale University Press, New Haven, Connecticut, p.455-505.

DUELLMAN, W.E. 1999. Distribution patterns of amphibians in South America. In Patterns of distribution of amphibians: a global perspective (W.E. Duellman, ed.). Johns Hopkins University Press, Baltimore, p.255-328.

DUELLMAN, W.E. 2005. Cusco Amazónico. The lives of amphibians and reptiles in an Amazonian rainforest. Comstock Publishing Associates, Cornell University Press, Ithaca. 
FEARNSIDE, P.M., ALENCASTRO-GRAÇA, P.M.L., KEIZER, E.W.H., MALDONADO, F.D., BARBOSA, R.I. \& NOGUEIRA, E.M. 2009. Modelagem de desmatamento e emissões de gases de efeito estufa na região sob influência da rodovia Manaus-Porto Velho (BR-319). Rev. Bras. de Meteorol. 24(2):208-233.

FRAGA, R., LIMA, A.P. \& MAGNUSSON, W.E. 2011. Mesoscale spatial ecology of a tropical snake assemblage: the width of riparian corridors in central Amazonia. Herpetol. J. 21:51-57.

FRANÇA, F.G.R. \& VENÂNCIO, N.M. 2010. Reptiles and amphibians of a poorly known region in southwest Amazonia. Biotemas. 23 (3):71-84.

FROST, D.R. 2011. Amphibian Species of the World: an Online Reference. version 5.5. American Museum of Natural History, New York. http://research.amnh.org/vz/herpetology/amphibia/ (último acesso em 14/02/2012).

GARDNER, T.A., RIBEIRO-JUNIOR, M.A., BARLOW, J., AVILA-PIRES, T.C.S., HOOGMOED, M.S. \& PERES, C.A. 2007. The value of primary, secondary and plantation forests for a neotropical herpetofauna. Conserv. Biol. 21:775-787. http://dx.doi.org/10.1111/j.1523-1739.2007.00659.x

GASCON, C. 1996. Amphibian litter fauna and river barriers in flooded and non-flooded Amazonian rainforests. Biotropica 28:136-140. http://dx.doi. org $/ 10.2307 / 2388779$

GASCON, C., MALCOLM, J.R., PATTON, J.L., SILVA, M.N.F., BOGART, J.P., LOUGHEED, S.C., PERES, C.A., NECKEL, S. \& BOAG, P. 2000. Riverine barriers in the geographic distribution of Amazonian species. P. Natl. Acad. Sci. 97(25):13672-13677. http://dx.doi.org/10.1073/ pnas.230136397

GORDO, M. 2003. Os anfíbios anuros do baixo Rio Purus/Solimões. In Piagaçu-Purus: Bases Científicas para Criação de uma Reserva de Desenvolvimento Sustentável (C. P. de Deus, R. Da Silveira \& L.H.R. Py-Daniel, eds.). IDSM, Manaus, p.65-71.

GOTELLI, N. J. \& COLWELL, R.K. 2001. Quantifying biodiversity: procedures and pitfalls in the measurement and comparison of species richness. Ecol. Lett. 4:379-391. http://dx.doi.org/10.1046/j.14610248.2001.00230.x

GOULDiNG, M., BARTHEM, R. \& FERREIRA, E.J.G. 2003. The Smithsonian Atlas of the Amazon. Smithsonian Institution Press, Washington.

GREENBERG, C.H., NEARY, D. \& HARRIS, L.D. 1994. A comparison of herpetofaunal sampling effectiveness of pitfall, single-ended, and doubleended funnel traps used with drift fences. J. Herpet. 28:319-324. http:// dx.doi.org/10.2307/1564530

HAUGAASEN, T. \& PERES, C.A. 2005. Mammal assemblage structure in Amazonian flooded and unflooded forests. J. Trop. Ecol. 21:133-145. http://dx.doi.org/10.1017/S026646740400207X

HAUGAASEN, T. \& PERES, C.A. 2006. Floristic, edaphic and structural characteristics of flooded and unflooded forests in the lower Rio Purús region of central Amazonia, Brazil. Acta Amaz. 36(1):25-36. http://dx.doi. org/10.1590/S0044-59672006000100005

HEYER, W.R. 1976. Notes on the frog fauna of the Amazon Basin. Acta Amaz. 6(3):369-378.

HEYER, W.R. 1977. Taxonomic notes on frogs from the Madeira and Purus Rivers, Brazil. Pap. Avulsos Zool. 31:141-162.

HESS, L.L., MELACK, J.M., NOVO, E., BARBOSA, C.C.F. \& GASTIL, M. 2003. Dual-season mapping of wetland inundation and vegetation for the central Amazon basin. Rem. Sens. Environ. 87:404-428. http:// dx.doi.org/10.1016/j.rse.2003.04.001

HOOGMOED, M.S. \& AVILA-PIRES, T.C.S. 2011. Sobre a presença de Scinax pedromedinae (Henle, 1991) (Amphibia: Anura: Hylidae) na Amazônia brasileira e norte do Peru. Bol. Mus. Para. Emilio Goeldi Cienc. Nat. 6(3):263-271.

INSTITUTOBRASILEIRODE GEOGRAFIAE ESTATÍSTICA-IBGE. 2002. Mapa de Climas do Brasil. IBGE, Brasil ftp://geoftp.ibge.gov.br/mapas/ tematicos/mapas_murais/clima.pdf/ (último acesso em 14/02/2012).
INSTITUTO PIAGAÇU (org.). 2010. Plano de Gestão da Reserva de Desenvolvimento Sustentável Piagaçu-Purus. Secretaria de Meio Ambiente e Desenvolvimento Sustentável, Governo do Amazonas, Manaus. http://www.ceuc.sds.am.gov.br/downloads/category/9planosdegestao.html (último acesso em 14/07/2011).

JUNK, W.J. 1997. The Central Amazon floodplains. Ecology of a pulsing system. Springer, Berlin, Heidelberg, New York. http://dx.doi. org/10.1007/978-3-662-03416-3

JUNK, W.J., BAYLEY, P.B., SPARKS, R.E. 1989. The flood pulse concept in river - floodplain systems. In: Proc Int Large River Symp (LARS) (D.P. Dodge ed.). Can. Spec. Publ. Fish. Aquat. Sci. 106:110-127.

LIMA, A.P., MAGNUSSON, W.E., MENIN, M., ERDTMANN, L.K., RODRIGUES, D.J., KELLER, C. \& HÖDL, W. 2008. Guia de Sapos da Reserva Adolpho Ducke: Amazônia Central. Attema Design Editorial Ltda, Manaus.

MARTINS JUNIOR, A.F. \& WAICHMAN, A.V. 2009. Mapeamento dos ambientes aquáticos e da cobertura da terra na Bacia do Rio Purus, Amazonas, Brasil. In Anais do XIV Simpósio Brasileiro de Sensoriamento Remoto (J.C.N. Epiphanio \& L.S. Galvão, eds.). Natal, v.1, p.5973-5980.

MARTINS, M. \& OLIVEIRA, M.E. 1998. Natural history of snakes in forests of the Manaus region, Central Amazonia, Brazil. Herpetol. Nat. Hist. 6(2):78-150.

MENIN, M., LIMA, A.P., MAGNUSSON, W.E \& WALDEZ, F. 2007. Topographic and edaphic effects on the distribution of terrestrially reproducing anurans in Central Amazonia: mesoscale spatial patterns. J. Trop. Ecol. 23:539-547. http://dx.doi.org/10.1017/S0266467407004269

MENIN, M., WALDEZ, F. \& LIMA, A.P. 2008. Temporal variation in the abundance and number of species of frogs in 10,000 ha of a forest in Central Amzonia, Brazil. South Am. J. Herpetol. 3:68-81. http://dx.doi. org/10.2994/1808-9798(2008)3[68:TVITAA]2.0.CO;2

MENIN, M., WALDEZ, F. \& LIMA, A.P. 2011. Effects of environmental and spatial factors on the distribution of anuran species with aquatic reproduction in central Amazonia. Herpetol. J. 21:255-261.

NECKEL-OLIVEIRA, S. \& GORDO, M. 2004. Anfíbios, lagartos e serpentes do Parque Nacional do Jaú. In Janelas para a biodiversidade no Parque Nacional do Jaú - uma estratégia para o estudo da biodiversidade na Amazônia (S.H. Borges, S. Iwanaga, C.C. Durigan \& M.R. Pinheiro, eds.). p.161-176.

O'HARA, R.B. 2005. Species richness estimators: how many species can dance on the head of a pin? J. Anim. Ecol. 74:375-386. http://dx.doi. org/10.1111/j.1365-2656.2005.00940.x

RIBEIRO-JÚNIOR, M.A., GARDNER, T.A. \& ÁVILA-PIRES, T.C.S. 2008. Evaluating the effectiveness of herpetofaunal sampling techniques across a gradient of habitat change in a tropical forest landscape. J. Herpet. 42:733. http://dx.doi.org/10.1670/07-097R3.1

RODRÍGUEZ, L.O. 2003. Anfibios y reptiles de la región del Alto Purús. In Alto Purús: Biodiversidad, Conservación y Manejo (R.L. Pitman, N Pitman \& P. Álvarez, eds). Gráfica S.A. Lima-Perú, Center for Tropical Conservation Duke University, p.89-96.

RODRÍGUEZ, L.O. \& DUELLMAN, W.E. 1994. Guide to the frogs of the Iquitos Region, Amazonian Peru. Univ. Kansas Mus. Nat. Hist. Spec. Publ. 22:1-80.

ROJAS-AHUMADA, D.P. \& MENIN, M. 2010. Composition and abundance of anurans in riparian and non-riparian areas in a forest in Central Amazonia, Brazil. South Am. J. Herpetol. 5:157-167. http://dx.doi. org/10.2994/057.005.0210

RON, S.R. 2000. Biogeographic area relationships of lowland Neotropical rainforest on raw distributions of vertebrate groups. Biol. J. Linn. Soc. 71:379-402. http://dx.doi.org/10.1111/j.1095-8312.2000.tb01265.x

SILVA, A.E.P., ANGELIS, C.F., MACHADO, L.A.T. \& WAICHAMAN, A.V. 2008. Influência da precipitação na qualidade da água do Rio Purus. Acta Amaz. 38(4):733-742. http://dx.doi.org/10.1590/S004459672008000400017

SILVA, J.M.C., RYLANDS, A.B. \& FONSECA, G.A.B. 2005. O destino das áreas de endemismo da Amazônia. Megadiversidade. 1(1):124-131. 
SOARES-FILHO, B.S., NEPSTAD, D., CURRAN, L., VOLL, E., CERQUeIRA, G., GARCIA, R.A., AZEVEDO-RAMOS, C., McDONALD, A., LEFEBVRE, P. \& SCHLESINGER, P. 2006. Modeling conservation in the Amazon basin. Nature. 440:520-523.

SOUZA, M. B. 2009. Anfíbios: Reserva Extrativista do Alto Juruá e Parque Nacional da Serra do Divisor, Acre. Editora do Instituto de Filosofia e Ciências Humanas - IFCH Unicamp, Campinas.

SOUZA, V.M., SOUZA, M.B. \& MORATO, E.F. 2008. Efeitos da sucessão florestal sobre a anurofauna (Amphibia: Anura) da Reserva Catuaba e seu entorno, Acre, Amazônia Sul - Ocidental. Rev. Bras. Zool. 25:49-57. http://dx.doi.org/10.1590/S0101-81752008000100008

TOCHER, M., GASCON, C. \& ZIMMERMAN, B.L. 1997. Fragmentation effects on a central Amazonian frog community: a ten-year study. In Tropical forest remnants: ecology, management, and conservation of fragmented communities (W.F. Laurance \& R.O. Bierregaard, eds.). University of Chicago Press, Chicago, p.124-137.

TURCI, L.C.B. \& BERNARDE, P.S. 2008. Levantamento herpetofaunístico em uma localidade no município de Cacoal, Rondônia, Brasil. Bioikos. 22:101-108.

VITT, L.J., AVILA-PIRES, T.C.S., CALDWELL, J.P. \& OLIVEIRA, V.R.L. 1998. The impact of individual tree harvesting on thermal environments of lizards in Amazonian rain forest. Conserv. Biol. 12:654-664. http://dx.doi.org/10.1046/j.1523-1739.1998.96407.x
VITT, L.J., MAGNUSSON, W.E., AVILA-PIRES, T.C.S. \& LIMA, A.P. 2008. Guia de Lagartos da Reserva Adolpho Ducke, Amazônia Central. Attema Design Editorial Ltda, Manaus.

VOGT, R.C., FERRARA, C.R., BERNHARD, R., CARVALHO, V.T., BALENSIEFER, D.C., BONORA, L. \& NOVELLE, S.M.H. 2007. Herpetofauna. In Biodiversidade do Médio Madeira: Bases científicas para propostas de conservação (L.R. Py-Daniel, C.P. Deus, A.L. Henriques, D.M. Pimpão, \& O.M. Ribeiro, eds). INPA, Manaus, p.127-143.

VOGT, R.C., MOREIRA, G. M. \& OLIVEIRA, C.D. 2001. Biodiversidade de répteis do bioma floresta amazônica e ações prioritárias para sua conservação. In Biodiversidade na Amazônia Brasileira (J.P.O.R. Capabianco, ed.). Estação Liberdade, Instituto Socioambiental, São Paulo, p.89-96.

WALDEZ, F. \& VOGT, R.C. 2007. Geographic Distribution. Cercosaura argulus (Elegant Eyed Lizard). Herpet. Rev. 37(4):493-494.

WALDEZ, F. \& VOGT, R.C. 2009. Aspectos ecológicos e epidemiológicos de acidentes ofídicos em comunidades ribeirinhas do baixo rio Purus, Amazonas, Brasil. Acta Amaz. 39(3):681-692. http://dx.doi.org/10.1590/ S0044-59672009000300025

WALDEZ, F. \& VOGT, R.C. 2011. As serpentes peçonhentas da Reserva Piagaçu-Purus e acidentes ofídicos na região do baixo rio Purus, Amazônia Central. Rev. Colombiana Cienc. Anim. 3:327-334.

ZIMMERMAN, B.L. 1994. Audio strip transects. In Measuring and monitoring biological diversity: standard methods for amphibians (W.R Heyer, M.A. Donnelly, R.W. McDiarmid, L.A.C. Hayek \& M.S. Foster, eds.). Smithsonian Institution Press, Washington, p.92-97.

Recebido em 10/04/2012

Versão reformulada recebida em 14/08/2012

Publicado em 11/03/2013 